\title{
The palladacycle complex AJ-5 induces apoptotic cell death while reducing autophagic flux in rhabdomyosarcoma cells
}

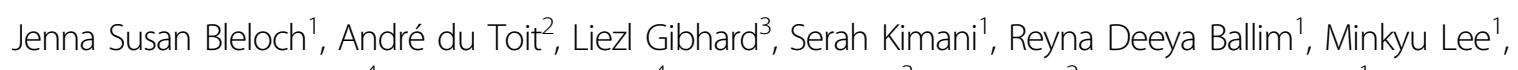
Angelique Blanckenberg ${ }^{4}$, Selwyn Mapolie $\mathbb{B}^{4}$, Lubbe Wiesner ${ }^{3}$, Ben Loos $^{2}$ and Sharon Prince ${ }^{1}$

\begin{abstract}
Rhabdomyosarcoma (RMS) forms in skeletal muscle and is the most common soft tissue sarcoma in children and adolescents. Current treatment is associated with debilitating side effects and treatment outcomes for patients with metastatic disease are dismal. Recently, a novel binuclear palladacycle, AJ-5, was shown to exert potent cytotoxicity in melanoma and breast cancer and to present with negligible adverse effects in mice. This study investigates the anticancer activity of AJ-5 in alveolar and embryonal RMS. IC $C_{50}$ values of $\leq 0.2 \mu \mathrm{M}$ were determined for AJ-5 and it displayed a favourable selectivity index of $>2$. Clonogenic and migration assays showed that AJ-5 inhibited the ability of RMS cells to survive and migrate, respectively. Western blotting revealed that AJ-5 induced levels of key DNA damage response proteins ( $\mathrm{YH} 2 \mathrm{AX}, \mathrm{p}-\mathrm{ATM}$ and p-Chk2) and the p38/MAPK stress pathway. This correlated with an upregulation of p21 and a $G_{1}$ cell cycle arrest. Annexin V-FITC/propidium iodide staining revealed that AJ-5 induced apoptosis and necrosis. Apoptosis was confirmed by the detection of cleaved PARP and increased levels and activity of cleaved caspases-3, -7, -8 and -9. Furthermore, AJ-5 reduced autophagic flux as shown by reduced LC3II accumulation in the presence of bafilomycin A1 and a significant reduction in autophagosome flux J. Finally, pharmacokinetic studies in mice show that AJ-5 has a promising half-life and that its volume of distribution is high, its clearance low and its intraperitoneal absorption is good. Together these findings suggest that AJ-5 may be an effective chemotherapeutic with a desirable mechanism of action for treating drug-resistant and advanced sarcomas.
\end{abstract}

\section{Introduction}

Rhabdomyosarcoma (RMS) originate in skeletal muscle and is the most common soft tissue sarcoma in children, adolescents and young adults ${ }^{1,2}$. It is classified as either embryonal (eRMS) or alveolar (aRMS), with each exhibiting distinct histological, molecular and pathological characteristics. Whereas eRMS affects predominantly

\footnotetext{
Correspondence: Sharon Prince (sharon.prince@uct.ac.za)

'Division of Cell Biology, Department of Human Biology, Faculty of Health Sciences, University of Cape Town, Cape Town, Western Cape, South Africa ${ }^{2}$ Department of Physiological Sciences, Stellenbosch University, Stellenbosch, Western Cape, South Africa

Full list of author information is available at the end of the article.
}

infants and children, aRMS occur primarily in adolescents and young adults. Furthermore, eRMS comprises approximately $67 \%$ of RMS cases with common sites of tumorigenesis including the head, neck and genitourinary system. On the other hand, aRMS accounts for approximately $30 \%$ of RMS cases with arms and legs often being sites of primary disease $\mathrm{e}^{3-5}$. RMS thus contributes to a considerable loss of years of life in comparison to other cancers as it largely affects children and adolescents.

The current standard treatment for patients with RMS is multimodal therapy consisting of local control with surgery and/or radiation therapy in conjunction with multi-agent chemotherapy ${ }^{6}$. Since the 1970s, disease 
control for patients with localized or completely resected RMS has been achieved with combinational chemotherapy most commonly including vincristine, dactinomycin (actinomycin D) and cyclophosphamide $(\mathrm{VAC})^{7}$. However, this treatment regimen has had limited success for patients with regional spreading, incomplete resection or metastasis $^{8}$. Furthermore, these drugs are associated with debilitating side effects including nausea, vomiting, fatigue, mouth ulcers, stomach ache, hair loss, bone marrow suppression, peripheral neuropathy and haemorrhagic cystitis $^{9-12}$. Continued collaborative efforts to find more effective treatments with fewer side effects is therefore important, especially for metastatic RMS.

The use of transition metal complexes as potential therapeutics and in diagnostic medicine is of considerable importance. Platinum drugs, particularly cisplatin, are the mainstay of metal-based compounds in the treatment of cancer. However, due to dose-related adverse effects and multi-drug resistance associated with this line of therapy there has been an ongoing search for alternative metallic compounds with improved anti-cancer and pharmacokinetic properties and distinct mechanisms of action ${ }^{13}$. Palladium-based complexes have recently been reported to exert significant cytotoxicity in cancer cells ${ }^{14}$. Importantly, studies have shown that while both platinum and palladium compounds induce DNA damage, the palladium compounds exert a much greater degree of cytotoxicity in cancer cells ${ }^{15,16}$. Moreover, numerous palladium complexes exhibit strong cytotoxic effects in cells resistant to cisplatin ${ }^{17}$. Together these results suggest that palladium complexes may exert cytotoxicity through a mechanism different to that of platinum compounds and that they may prove to be less toxic and more effective for the treatment of aggressive cancers. Recently, AJ-5, a novel binuclear palladacycle complex with 1,2-bis(diphenylphosphino)ethane as co-ligand was shown to exert potent anti-tumour activity in advanced melanoma and breast cancer cells with very promising in vivo clearance of advanced melanoma ${ }^{18,19}$. In the present study we therefore explored these questions in RMS and other sarcoma subtypes. We show here for the first time that AJ-5 displays potent and selective cytotoxicity at sub-micromolar concentrations $(\leq 0.2 \mu \mathrm{M})$ against aRMS and eRMS cells and inhibits their ability to survive, proliferate and migrate. We show that AJ-5 induces double-stranded DNA breaks (DSBs) which triggers the canonical DSBs ataxia telangiectasia mutated serine/threonine kinase (ATM)/checkpoint kinase 2 (Chk2) pathway as well as the p38/mitogen-activated protein kinase (MAPK) stress signalling pathway leading to a $G_{1}$ cell cycle arrest, reduction in autophagic flux and induction of apoptosis. Furthermore, AJ-5 shows efficacy in a range of sarcoma subtypes and displays a promising pharmacokinetic profile in healthy mice. This study provides evidence that AJ-5 has promising therapeutic potential for the treatment of RMS and a range of other sarcoma subtypes.

\section{Results \\ AJ-5 shows potent and selective cytotoxicity against RMS cells}

Here we investigated the anti-tumour effects of the binuclear palladacycle complex, AJ-5, in RMS cells. To this end, MTT assays were performed on aRMS (RH30 and AX-OH-1 cell lines) and eRMS (RD and FL-OH-1 cell lines) cells treated with a range of AJ-5 concentrations $(0.1-1.0 \mu \mathrm{M})$ for $48 \mathrm{~h}$. IC $\mathrm{I}_{50}$ concentrations of $\leq 0.2 \mu \mathrm{M}$ were obtained for all cell lines (Fig. 1a). Light microscopy images of RMS cells treated for 24 and $48 \mathrm{~h}$ with their respective $\mathrm{IC}_{50}$ concentrations show the impact of AJ-5 on cell viability and morphology (Fig. 1b). To determine the selectivity of $\mathrm{AJ}-5$ for RMS cells, $\mathrm{IC}_{50}$ values were determined in non-malignant fibroblasts (FG0 and $\mathrm{DMB}$ ) and mouse myoblasts (C2C12). Results show that AJ-5 is less cytotoxic in the non-malignant cells with a selectivity index (SI) of $>2$ in all cases (Fig. 1c). Interestingly, there is growing evidence that mesenchymal stem cells are the cell of origin for specific sarcoma types including $\mathrm{RMS}^{20,21}$, and compared to the other non-malignant cells tested, the mesenchymal stem cells (A100021501) were the most sensitive to AJ-5.

\section{AJ-5 inhibits the ability of RMS cells to survive, proliferate and migrate}

To explore the potential impact of AJ-5 on the longterm fate of RMS cells, clonogenic assays were performed $^{22}$. Figure $2 \mathrm{a}$ shows representative images and quantification of colonies formed over 7-21 days after 24 $\mathrm{h}$ of AJ-5 treatment. It is evident that AJ-5 significantly inhibits the ability of RMS cells to survive and proliferate. Indeed, for all except one RMS cell line tested, there was a significant decrease in colony area at $1 / 4 \mathrm{IC}_{50}$ treatment and only a few or no RMS cell colonies formed at $\mathrm{IC}_{50}$ concentrations. Importantly, the ability of the nonmalignant $\mathrm{C} 2 \mathrm{C} 12$ myoblasts to survive and proliferate was not significantly hindered after $24 \mathrm{~h}$ of $0.3 \mu \mathrm{M} \mathrm{AJ}-5$ (Fig. 2b). This is at least 1.5 times higher than the $\mathrm{IC}_{50}$ values obtained for the RMS cells. To further explore the anti-cancer activity of AJ-5, RMS cells were exposed for $24 \mathrm{~h}$ to $\mathrm{IC}_{50}$ concentrations of the drug and scratch motility assays were performed. Fig. 2c shows that AJ-5 reduced the migratory ability of all RMS cells tested. Taken together, these data suggest that AJ-5 inhibits the ability of RMS cells to survive, proliferate and migrate.

To further explore the mechanisms by which AJ-5 exerts its cytotoxicity, the aRMS cell line RH30 and the eRMS cell line RD were used for further characterization. 


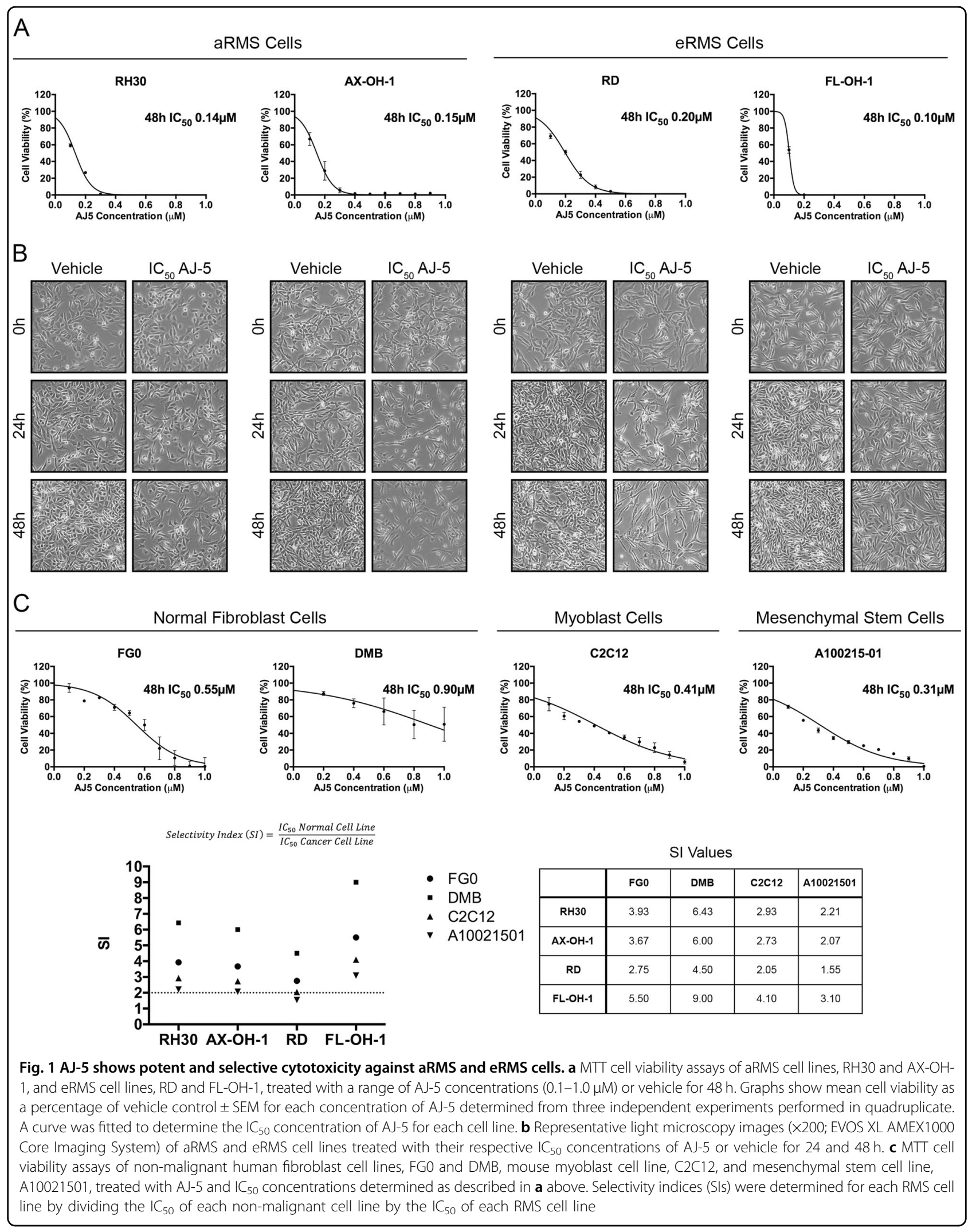


A
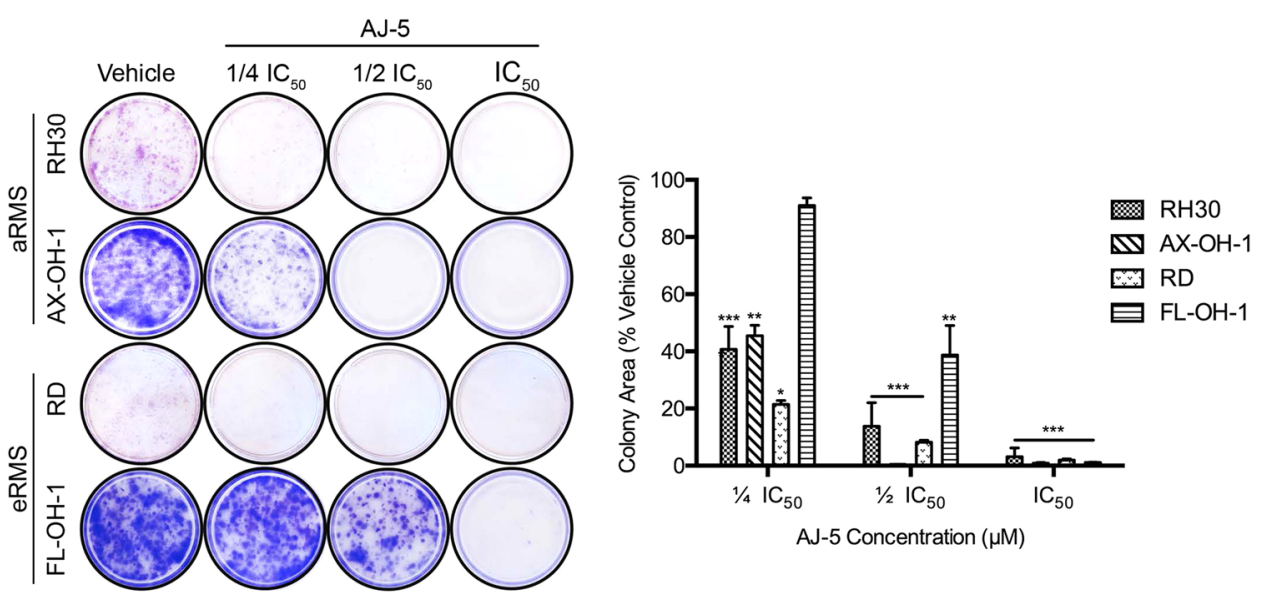

B
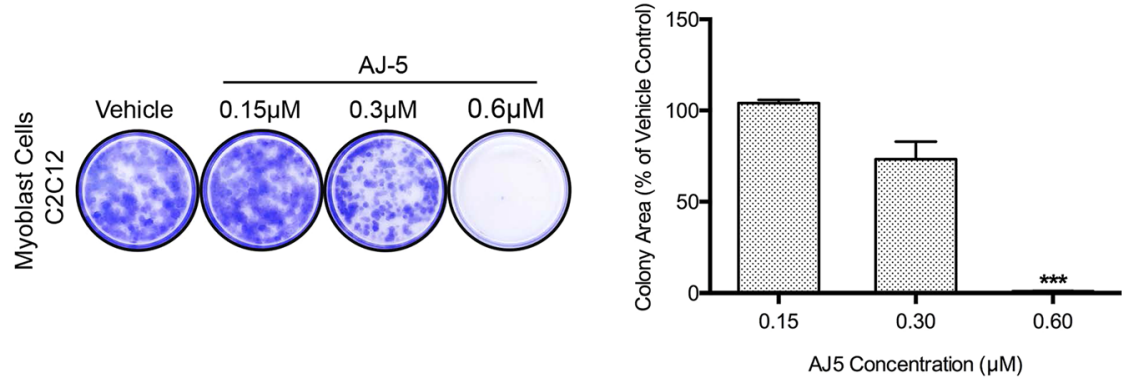

C aRMS
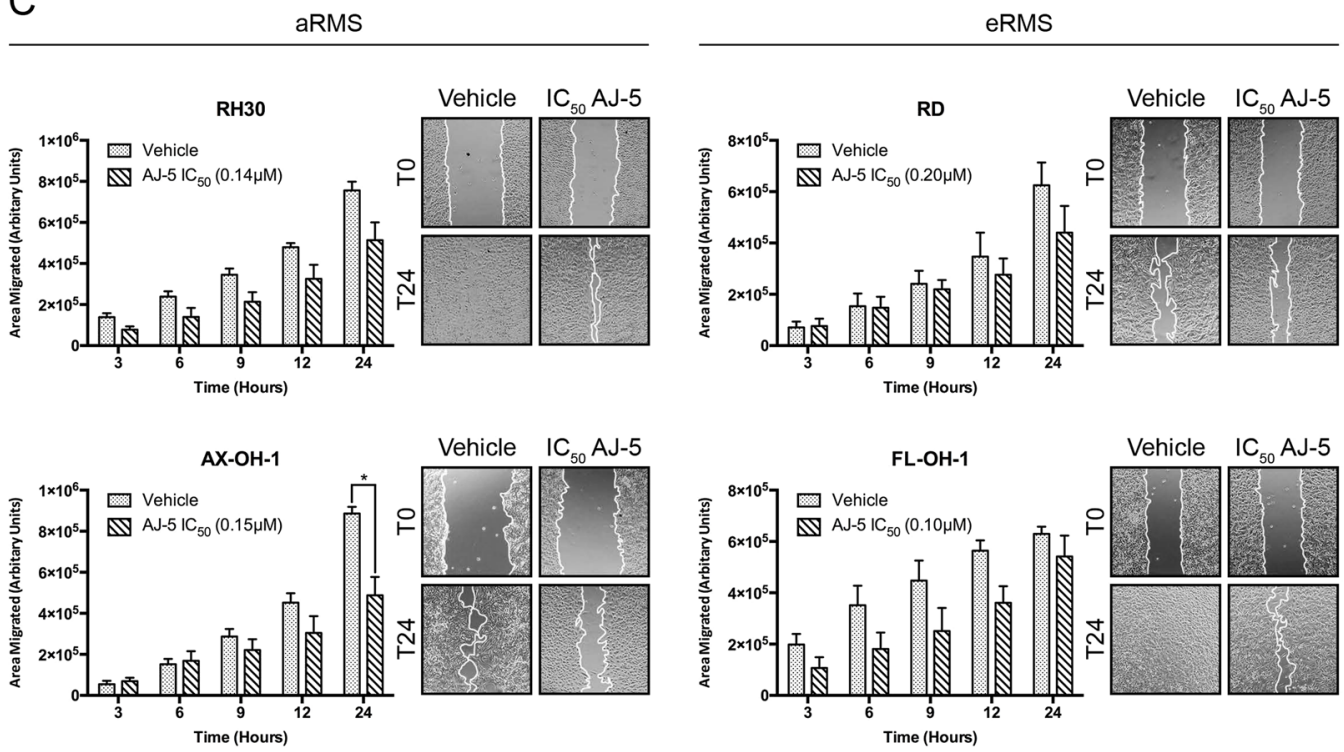

Fig. 2 AJ-5 inhibits the ability of RMS cells to survive, proliferate and migrate. a Representative images and quantification of clonogenic assays of eRMS and aRMS cells treated with vehicle, $1 / 4, I_{50}, 1 / 2, C_{50}$ or IC $C_{50}$ concentrations of AJ-5 for $24 \mathrm{~h}$ and then replated at low densities in drug-free medium and left for 7-21 days for colonies to form. Colonies were stained with crystal violet and images from three independent repeats were quantified using the ImageJ plugin ColonyArea. The graph represents the mean colony area \pm SEM of each treatment condition as a percentage of the vehicle control. $\mathbf{b}$ The same as $\mathbf{a}$ above for the non-malignant mouse myoblast cell line C2C12, but treated with vehicle, $0.15 \mu \mathrm{M}, 0.3 \mu \mathrm{M}$, or 0.6 uM AJ-5. c Representative images ( $\times 200$; EVOS XL AMEX1000 Core Imaging System) and quantification of scratch motility assays of eRMS and aRMS cells pre-treated with $\mathrm{IC}_{50}$ concentrations of AJ-5 or vehicle for $24 \mathrm{~h}$ and then replated at $100 \%$ confluency in drug-free medium. After cell adherence, a sterile $2 \mu \mathrm{L}$ pipette tip was used to make a linear wound in the cell monolayer and cells were treated with $10 \mu \mathrm{g} / \mathrm{mL}$ mitomycin $C$ to inhibit proliferation. Cells were imaged at 0, 3, 6, 9, 12 and $24 \mathrm{~h}$ post wound formation. Total area migrated was calculated by subtracting the wound area at each time point from the wound area at time $0 \mathrm{~h}$, which is represented in the graphs as mean area migrated \pm SEM pooled from three independent repeats. Data were analysed using GraphPad Prism 6.0 and a parametric unpaired $t$-test was performed where ${ }^{*} p<0.05,{ }^{* *} p<0.01,{ }^{* * *} p<0.001$ 


\section{AJ-5 activates the DNA damage and p38 MAPK pathways in RMS cells}

$\gamma \mathrm{H} 2 \mathrm{AX}$ is a robust marker of $\mathrm{DSBs}^{23}$ and western blotting and immunocytochemistry revealed a dosedependent increase in levels of $\gamma \mathrm{H} 2 \mathrm{AX}$ (Fig. 3a) and an accumulation of distinct $\gamma \mathrm{H} 2 \mathrm{AX}$ foci in the nuclei of AJ5-treated cells (Fig. 3b), respectively. Furthermore, AJ-5 treatment led to increased levels of phosphorylated ATM and its downstream target CHK2 as well as phosphorylated p38 (Fig. 3c). AJ-5 thus activated the canonical DSB and p38 MAPK stress signalling pathways. Both of these pathways converge on $\mathrm{p} 53$, which ordinarily plays an important role in transcriptionally activating the cyclindependent kinase $\mathrm{p} 21^{24}$. However, both the RMS cell lines used in this study harbour p53 mutations that result in high levels of inactive $\mathrm{p} 53^{25}$. Importantly, p21 levels increased at the $\mathrm{IC}_{50}$ concentrations of $\mathrm{AJ}-5$ and this response was particularly pronounced after $24 \mathrm{~h}$ of treatment (Fig. 3c). These results suggest that p21 levels increase in a p53-independent manner.

\section{AJ-5 triggers a $G_{1}$ cell cycle arrest and induces apoptosis and necrosis}

To investigate the effect of AJ-5 on cell cycle progression, FACS analyses were performed on RMS cells treated with drug for 24 and $48 \mathrm{~h}$. Results show that AJ-5 induces a $G_{1}$ cell cycle arrest in both cell lines at the expense of the $S$ phase (Fig. 4a). This correlated with a decrease in levels of cyclin $\mathrm{A}$ and cyclin $\mathrm{B}$, which are required for progression through the $S$ and $G_{2} / M$ phases, respectively $^{26}$ (Fig. 4b). The sub- $\mathrm{G}_{1}$ peaks seen in Fig. 4a suggested that AJ-5 treatment leads to cell death. Indeed, Annexin V-FITC assays confirm that AJ-5 induces apoptotic and necrotic cell death (Fig. 4c).

\section{AJ-5 triggers the intrinsic and extrinsic apoptosis pathways in RMS cells}

To confirm that AJ-5-induces apoptosis, RD and RH30 cells were treated with AJ-5 and biological, molecular and biochemical markers of apoptosis were investigated by light microscopy, western blotting and enzymatic Caspase-Glo assays, respectively. The results show that AJ-5 induced characteristic features of apoptosis including membrane blebbing and cell shrinkage (Fig. 5a). In addition, AJ-5 treatment led to increased levels and activity of cleaved caspase- 8 and caspase- 9 , the effectors of the extrinsic and intrinsic pathways, respectively (Fig. 5b, c). This correlated with increased levels of the active (cleaved) forms of the executioner caspases-3/7 and their substrate poly (ADP-ribose) polymerase (PARP) (Fig. 5b, c c) ${ }^{27,28}$. Interestingly, we observed an $\sim 20 \mathrm{kDa}$ band above the $18 \mathrm{kDa}$ active cleaved caspase- 8 fragment. A similar band has also been reported by other researchers and it has been suggested that it represents an intermediate product of procaspase-8 processing ${ }^{29,30}$. Importantly, caspases- 8 and -9 were more robustly activated in RD cells treated with AJ-5 than those treated with $\mathrm{IC}_{50}$ doxorubicin, a drug commonly used in the treatment of RMS and used as a positive control in apoptosis assays (Fig. 5c). Together these results show that AJ-5 induces cell death in RMS cells, in part, through the extrinsic and intrinsic apoptotic pathways.

\section{AJ-5 induces several markers of autophagy in RMS cells}

We next investigated whether AJ-5 induced autophagy because large vacuolar structures and acidic vesicles were observed in RMS cells treated with this drug (Supplementary Fig. S1A). To this end, western blotting and immunocytochemistry were performed with an antibody to microtubule-associated protein light chain 3 (LC3). Supplementary Figure S1B shows that LC3II (the autophagosomal membrane bound form of LC3) was induced in both RMS cell lines in a time- and dose-dependent manner. Furthermore, supplementary Fig. S1C shows distinct puncta, presumed to represent LC3II aggregates conjugated to autophagosomal membranes, in RD and RH30 cells from as early as $6 \mathrm{~h}$ after AJ- 5 treatment.

\section{AJ-5 reduces autophagic flux in RD and RH30 cells}

The detection of LC3II alone does not distinguish between autophagy induction and cessation in the downstream steps of autophagy. We therefore next determined the impact of AJ-5 treatment on autophagic flux by measuring the levels of p62, a receptor of polyubiquitinated proteins. The rationale for this is based on observations that upon autophagy induction, p62 is incorporated within an autophagosome and degraded within an autolysosome ${ }^{31}$. The turnover of p62 is therefore considered an indicator of autophagic flux. Interestingly, Fig. 6a shows that whereas AJ-5 appears to reduce autophagic flux in RH30 cells, it increases autophagic flux in RD cells. To confirm the above data, levels of LC3II were determined by western blotting in the presence and absence of bafilomycin A1 which blocks the autophagy pathway by inhibiting fusion of autophagosomes with lysosomes $^{32}$. An accumulation of LC3II in the presence of this inhibitor is therefore considered evidence of autophagic flux. Consistent with our p62 data in RH30 cells, AJ5 treatment followed by bafilomycin A1 exposure led to decreased levels of LC3II (Fig. 6b). Interestingly, contrary to our p62 data in RD cells, in the presence of bafilomycin A1, AJ-5 treatment led to a negligible change in LC3II compared with vehicletreated cells (Fig. 6b). We therefore further investigated the impact of AJ-5 on autophagic flux in the RD cell line by performing single-cell autophagosome flux analyses. Our results reveal that RD cells are characterized by a predominant autolysosomal pool ( $n A=83$ autolysosomes/cell), with only a very minor pool 
A

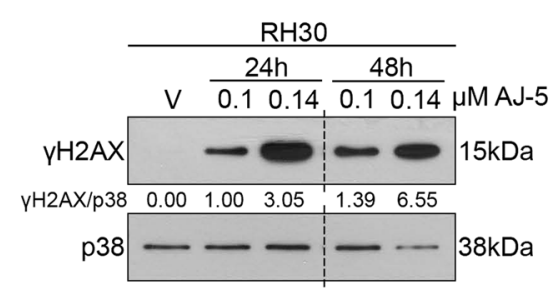

$\mathrm{B}$

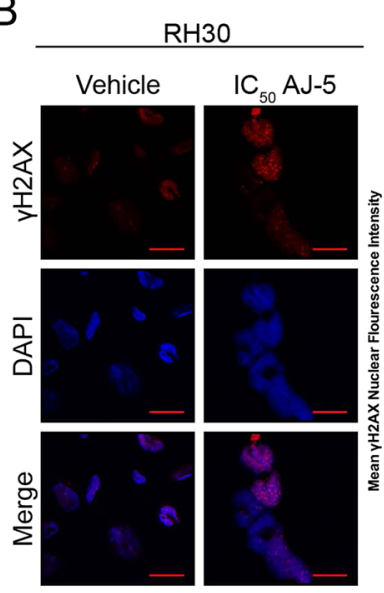

24h Treatment

C

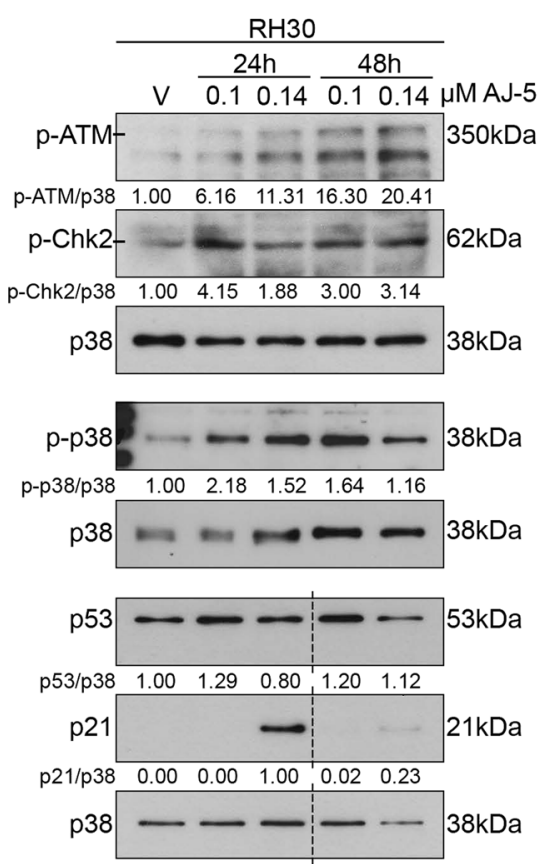

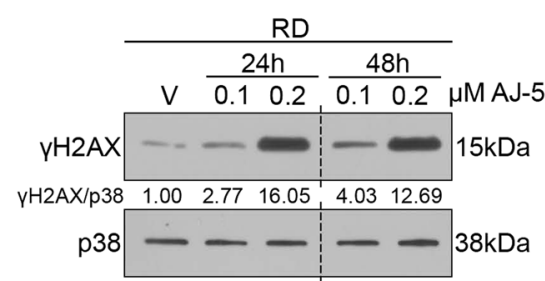

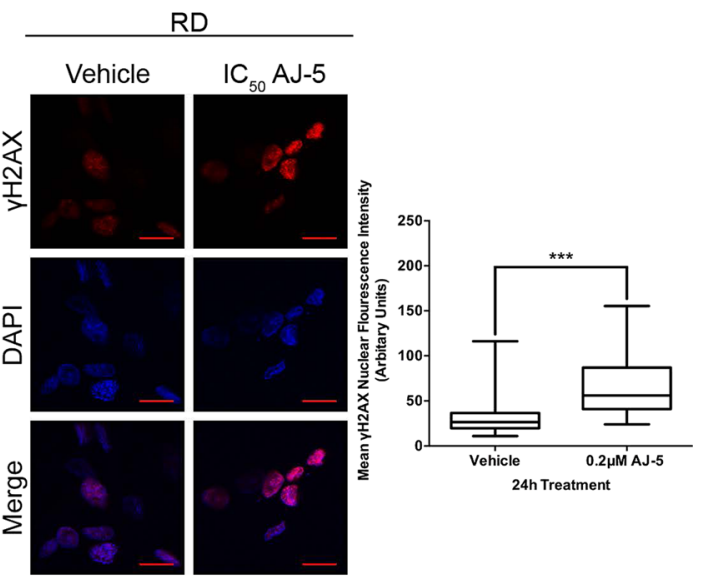

24h Treatment

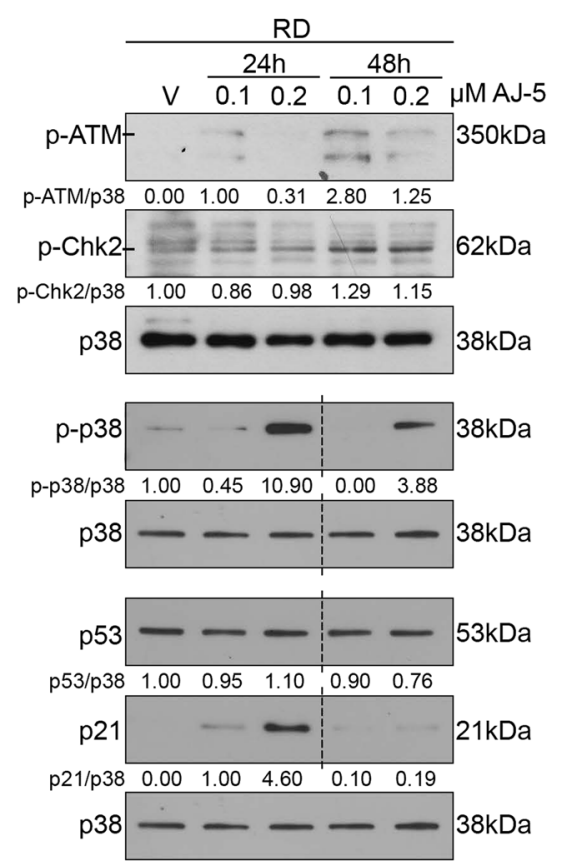

Fig. 3 AJ-5 activates the DNA damage and the p38 MAPK pathways. a $\gamma \mathrm{H} 2 \mathrm{AX}$ protein levels detected by western blotting in RH30 (aRMS) and $\mathrm{RD}$ (eRMS) cells treated with vehicle $(\mathrm{V}), 0.1 \mu \mathrm{M}$ or $\mathrm{IC}_{50} \mathrm{AJ}-5$ for 24 and $48 \mathrm{~h}$. p38 was used as a loading control. Densitometry readings were obtained using ImageJ and protein expression levels are represented as a ratio of protein of interest/p38 normalized to the vehicle control sample (where possible). Blots are representative of at least two independent repeats. $\mathbf{b}$ Representative confocal immunofluorescence maximum intensity projection images ( $\times 630$; Carl Zeiss LSM 510) of RH30 and RD cells treated with IC 50 AJ-5 or vehicle for $24 \mathrm{~h}$ and $\gamma \mathrm{H} 2 \mathrm{AX}$ detected with a fluorophore-conjugated Cy3 secondary antibody. Nuclei of cells were stained with DAPI. Scale bar is $20 \mu \mathrm{M}$. Box plots represent quantification of $y \mathrm{H} 2 \mathrm{AX}$ levels per treatment condition as mean nuclear Cy3 fluorescence from 20 fields of view from three independent repeats. Data were analysed using GraphPad Prism 6.0 and a parametric unpaired $t$-test was performed where ${ }^{*} p<0.05,{ }^{* *} p<0.01,{ }^{* * *} p<0.001$. c Western blot analyses with antibodies to key DNA damage and stress signalling pathway proteins: p-ATM, p-Chk2, p-p38, p53, and p21. RH30 and RD cells were treated with AJ-5 and protein expression quantified as described above in $\mathbf{a}$. Broken lines in western blots shown in this figure indicate where lanes not relevant were removed 
A
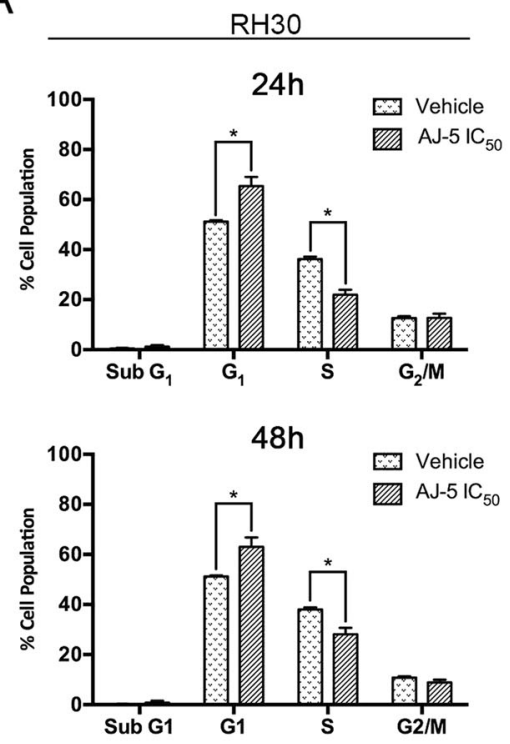

B
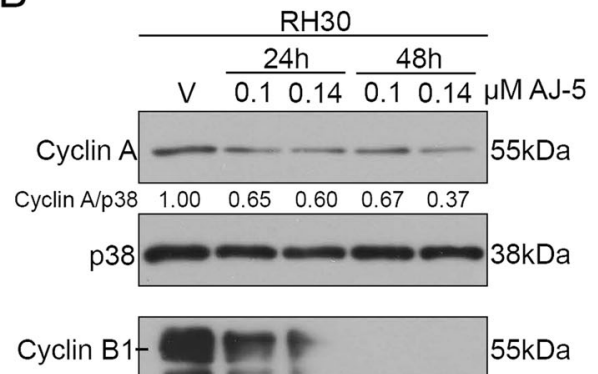

\begin{tabular}{llllll} 
Cyclin B1/p38 & 1.00 & 0.90 & 0.31 & 0.00 & 0.00 \\
\hline
\end{tabular}

p38

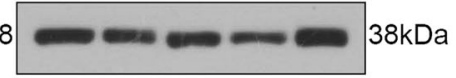

C

$\mathrm{RH} 30$
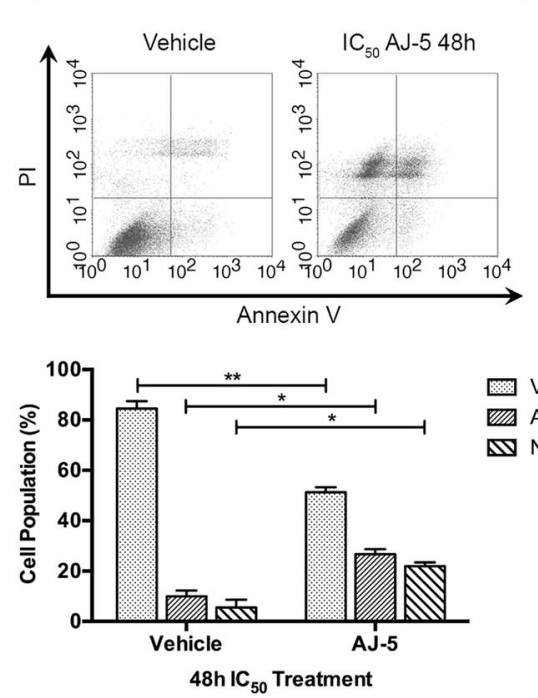
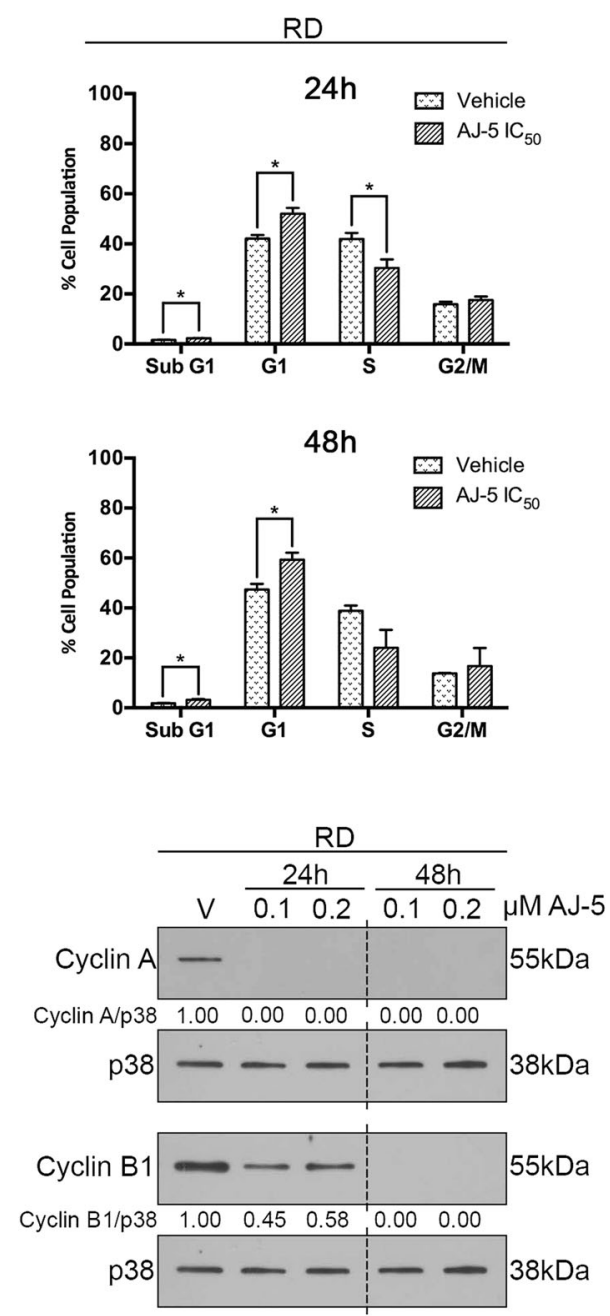

RD
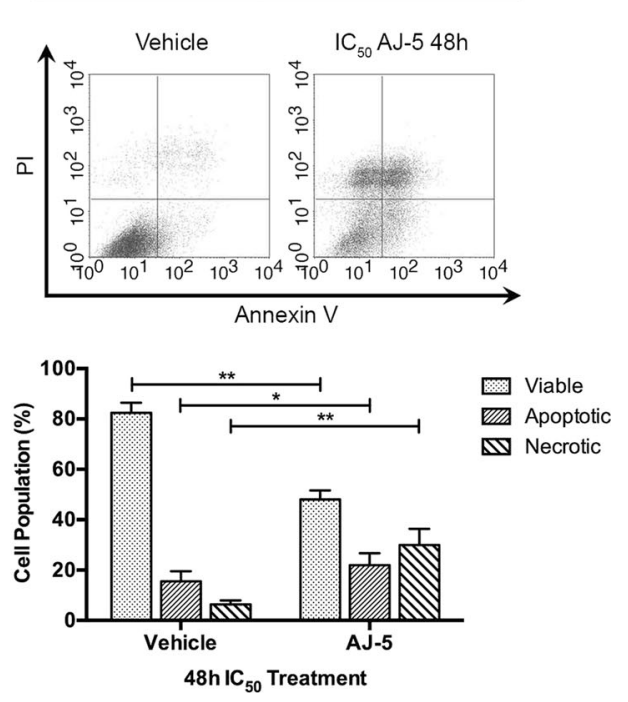

Fig. 4 (See legend on next page.) 
(see figure on previous page)

Fig. 4 AJ-5 triggers a G1 cell cycle arrest and induces apoptotic and necrotic cell death in RH30 and RD cells. a Flow cytometry analyses of cells treated with vehicle or $\mathrm{IC}_{50}$ AJ-5 for 24 and $48 \mathrm{~h}$. Graphs represent the mean proportion of cells \pm SEM at each phase of the cell cycle pooled from three independent repeats. $\mathbf{b}$ Western blot analyses of protein harvested from cells treated as indicated and incubated with antibodies against cell cycle markers cyclin A and cyclin B1. p38 was used as a loading control. Densitometry readings were obtained using ImageJ and protein expression levels are represented as a ratio of protein of interest/p38 normalized to the vehicle control sample. Blots are representative of at least two independent repeats. Broken lines indicate where lanes not relevant were removed. c Flow cytometric analyses of cells treated with vehicle or $I_{50}$ AJ-5 and stained with Annexin V-FITC and PI. Graphs represent the mean \pm SEM percentage of viable (lower left-hand quadrant), apoptotic (lower right-hand quadrant + upper right-hand quadrant) and necrotic (upper left-hand quadrant) cells from three independent experiments. For $\mathbf{a}$ and $\mathbf{c}$ data were analysed using GraphPad Prism 6.0 and a parametric unpaired $t$-test was performed where ${ }^{*} p<0.05,{ }^{* *} p<0.01,{ }^{* * *} p<0.001$

of autophagosomes and lysosomes per cell (Fig. 6c). Moreover, the cells are characterized by a basal autophagosome flux $J$ of 6.3 autophagosomes per hour per cell. Upon AJ-5 treatment, however, both autolysosome pool size as well as autophagosome flux significantly decreased. This suggests that AJ-5 negatively impacts the rate of autophagosome synthesis, which supports the data showing that in the presence of bafilomycin A1, AJ-5 treatment does not lead to LC3II accumulation (Fig. 6b). Together these data suggest that AJ-5 reduces autophagic flux in RH30 and RD cells.

\section{AJ-5 is cytotoxic in a range of sarcoma subtypes}

To investigate if the therapeutic potential of AJ-5 could be extended to other sarcoma subtypes, chondrosarcoma (SW1353), liposarcoma (SW872), synovial sarcoma (SW982), fibrosarcoma (HT1080) and osteosarcoma (MG-63) cells were treated with the drug as described earlier and MTT assays were performed. Our results show that an $\mathrm{IC}_{50}$ of $<0.3 \mu \mathrm{M}$ was obtained for all the sarcoma cell lines tested (Supplementary Fig. S2A) and a favourable SI of $>2$ was achieved when calculated relative to the combined $\mathrm{IC}_{50}$ values for the normal fibroblasts (FG0 and DMB). However, a sub-optimal SI between 1 and 1.5 was obtained when the $\mathrm{IC}_{50}$ values for the sarcoma cells were expressed relative to the mesenchymal stem cells (A10021501) (Supplementary Fig. S2B). This raises the interesting possibility that AJ-5 may be effective against the cells of origin of these sarcoma subtypes which may be of therapeutic benefit. Furthermore, clonogenic assays reveal that as little as a $1 / 4 \mathrm{IC}_{50}$ concentration of $\mathrm{AJ}-5$ significantly reduced the ability of cells of all sarcoma subtypes to survive and proliferate (supplementary Fig. S2C). AJ-5 therefore shows potent selective cytotoxicity against a number of diverse sarcoma subtypes and may therefore have broad therapeutic potential.

\section{Pharmacokinetic (PK) profile of AJ-5 in healthy mice}

Given its importance to the drug discovery process, we next tested the in vivo PK profile of AJ-5 in whole blood of MF1 mice following a single dose of $2 \mathrm{mg} / \mathrm{kg}$ intravenous (IV), $2 \mathrm{mg} / \mathrm{kg}$ intraperitoneal (IP) or $20 \mathrm{mg} / \mathrm{kg}$ oral (PO) for a period of $24 \mathrm{~h}$. The blood concentration-time curve of AJ-5 over a $24 \mathrm{~h}$ period and the calculated PK parameters are shown in Supplementary Fig. S3 and Table S1. For IV administration, AJ-5 illustrated a long half-life $(>10 \mathrm{~h})$, which is most likely due to the low clearance $(9.2$ $\mathrm{mL} / \mathrm{min} / \mathrm{kg})$ and a high volume of distribution $(8.8 \mathrm{~L} / \mathrm{kg})$. The exposure of AJ-5 following the IP dose of $2 \mathrm{mg} / \mathrm{kg}$ was eight-fold higher compared to the PO dose of $20 \mathrm{mg} /$ $\mathrm{kg}$ with an area under the curve of 88 and $11 \mathrm{~min} . \mu \mathrm{M} / \mathrm{L}$, respectively. The data obtained for the IP group in healthy mice correlated well with our previously observed in vivo efficacy of AJ-5 in advanced melanoma ${ }^{18}$.

\section{Discussion}

RMS is the most common soft tissue sarcoma found in children and adolescents and while the current treatment for localized tumours results in a high overall survival rate, the chemotherapeutic agents used are associated with debilitating adverse effects ${ }^{10,33-36}$. Moreover, more than $15 \%$ of patients present with metastatic disease and there has been limited improvement in the treatment of patients with recurrent and metastatic disease ${ }^{37-41}$. The current study provides several lines of evidence that the binuclear palladacycle AJ-5 may be a promising chemotherapeutic to treat aRMS and eRMS cells as well as a range of other sarcoma subtypes. Furthermore, we provide novel and interesting mechanisms by which AJ-5 functions (see Fig. 7).

Platinum compounds such as cisplatin have been used successfully as anti-tumour agents but they have been associated with severe toxicity and tumour drug resistance $^{42}$. Palladium compounds have been proposed as more effective alternatives because they appear to be more active at lower concentrations with less severe side effects $^{43,44}$. Cisplatin is occasionally used to treat RMS $^{6}$ and was reported to have an $\mathrm{IC}_{50}$ of $>0.5 \mu \mathrm{M}$ after 4-5 days of treatment in a range of RMS cell lines including RD and RH30 cells ${ }^{45,46}$. Our study shows that after only 2 days of AJ- 5 treatment, $\mathrm{IC}_{50}$ values of $\leq 0.2$ $\mu \mathrm{M}$ were obtained in the RD and RH30 cells. This suggests that AJ-5 displays more potent anti-cancer activity at much lower concentrations over a shorter 
A

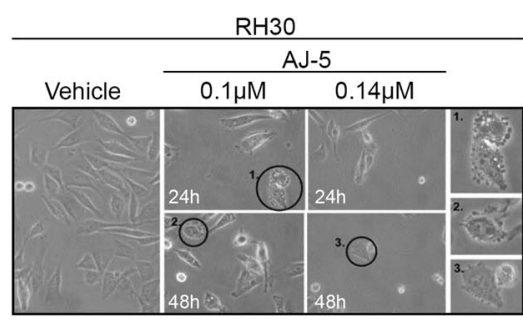

B
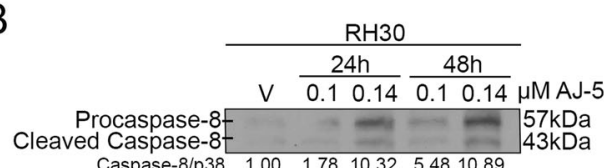

$\begin{array}{cccccc}\text { Caspase-8/p38 } & 1.00 & 1.78 & 10.32 & 5.48 & 10.89 \\ \text { Cleaved Caspase-8/p38 } & 1.00 & 1.77 & 6.00 & 2.92 & 5.88\end{array}$

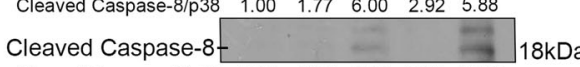

$\begin{array}{llllll}\text { Cleaved Caspase-8/p38 } & 1.00 & 2.09 & 6.33 & 0.90 & 16.01\end{array}$

$$
\text { p38 }=38 \mathrm{kDa}
$$

Procaspase-9 $=-m 47 \mathrm{kDa}$

Cleaved Caspase-9f $35 / 37 \mathrm{kDa}$

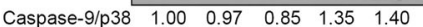

Cleaved Caspase-9 p37/p38 $1.00 \quad 1.38 \quad 1.34 \quad 2.34 \quad 8.13$

$\begin{array}{llllllll}\text { Cleaved Caspase-9 p35/p38 } & 1.00 & 1.40 & 2.07 & 1.06 & 33.22\end{array}$
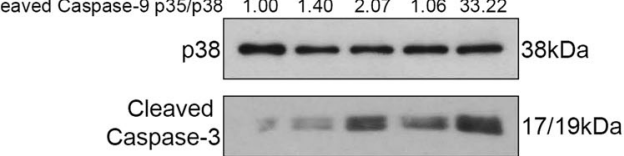

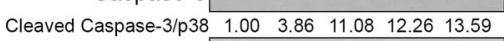

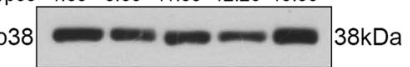

Cleaved $18 \mathrm{kDa}$

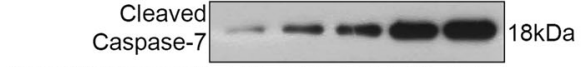

Cleaved Caspase-7/p38 $1.00 \quad 5.88 \quad 7.05 \quad 10.01 \quad 12.03$
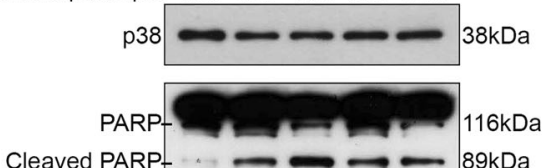

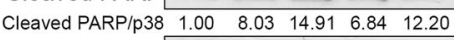

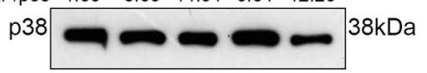

C

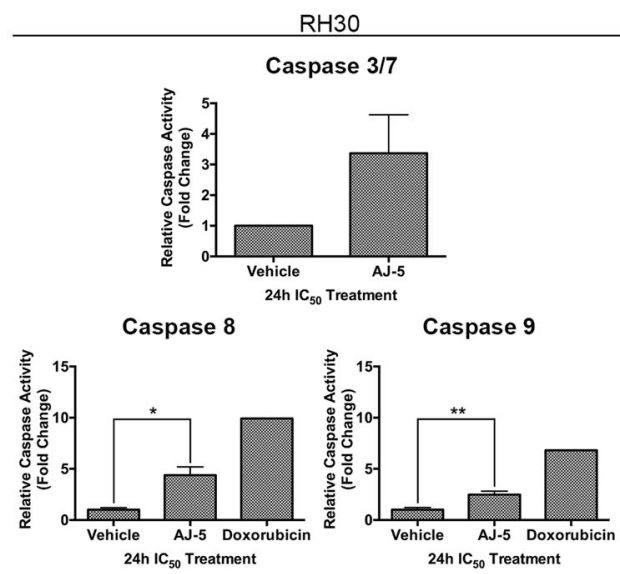

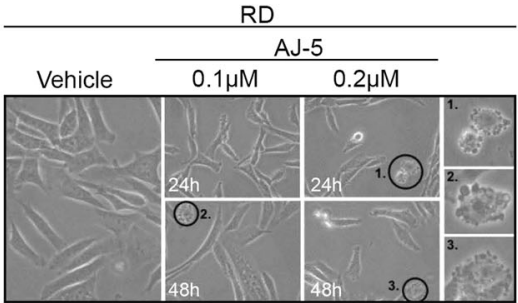

$$
\begin{gathered}
\frac{\mathrm{RD}}{\mathrm{n}} \\
\mathrm{n} \frac{24 \mathrm{~h}}{0.10 .2} \frac{48 \mathrm{~h}}{0.10 .2} \mu \mathrm{M} \mathrm{AJ}-5
\end{gathered}
$$

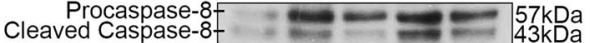
\begin{tabular}{ccccccc}
\hline Caspase-8/p38 & 1.00 & 7.40 & 3.75 & 4.33 & 3.06 \\
\hline
\end{tabular} $\begin{array}{llllll}\text { Cleaved Caspase-8/p38 } & 1.00 & 3.68 & 0.82 & 3.85 & 1.68\end{array}$ Cleaved Caspase-8- $\quad=18 \mathrm{kDa}$ $\begin{array}{llllll}\text { Cleaved Caspase-8/p38 } & 0.00 & 0.00 & 1.00 & 5.16 & 3.34\end{array}$

$$
\mathrm{p} 38=38 \mathrm{kDa}
$$

Procaspase-9- $47 \mathrm{kDa}$

Cleaved Caspase-9= $35 / 37 \mathrm{kDa}$

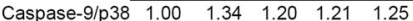

$\begin{array}{llllll}\text { Cleaved Caspase-9 p37/p38 } & 0.00 & 1.00 & 2.53 & 3.36 & 16.77 \\ \text { Cleaved Caspase-9 p35/p38 } & 1.00 & 3.22 & 6.73 & 5.91 & 15.49\end{array}$

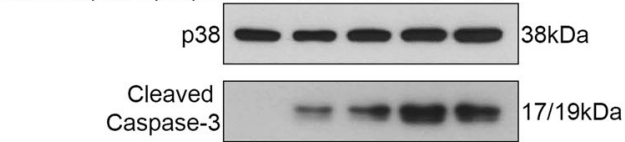

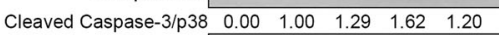

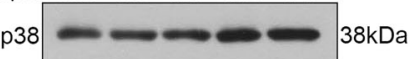

Cleaved $\longrightarrow 0.00$

Caspase-7 $\rightarrow-m=18 \mathrm{kDa}$

Cleaved Caspase-7/p38 $1.00 \quad 2.08 \quad 2.60 \quad 4.35 \quad 3.56$
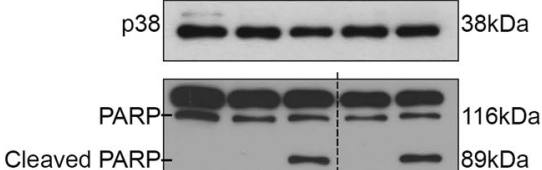

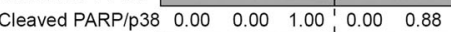
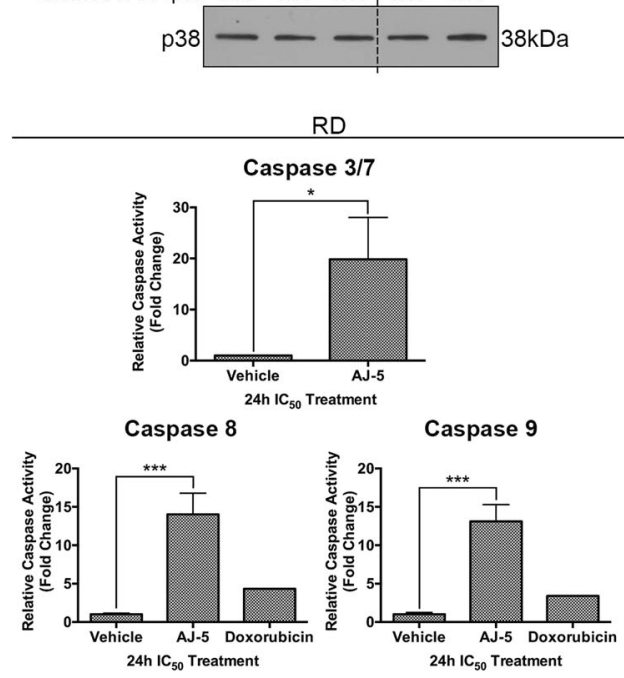

Fig. 5 (See legend on next page.) 
(see figure on previous page)

Fig. 5 AJ-5 triggers the intrinsic and extrinsic apoptosis pathways in RMS cells. a Representative light microcopy images ( $\times 200$; EVOS XL AMEX1000 Core Imaging System) showing the morphology of RH30 and RD cells treated with vehicle, $0.1 \mu \mathrm{M}$ or IC $\mathrm{C}_{50}$ AJ-5 for 24 and 48 h. Numbered circles correspond to magnified images on the right, which highlight characteristic apoptotic morphology including membrane blebbing and cell shrinkage. $\mathbf{b}$ Western blot analyses of protein harvested from cells treated as in $\mathbf{a}$ and incubated with antibodies as indicated. p38 was used as a loading control and densitometry readings were obtained using ImageJ. Protein expression levels are represented as a ratio of protein of interest/p38 normalized to vehicle control samples (where possible). Blots are representative of at least two independent repeats. Broken lines indicate where lanes not relevant were removed. c Caspase-Glo assays showing the enzymatic activity of caspase-3/7, caspase-8 and caspase-9 for cells treated with vehicle or $\mathrm{I}_{50} \mathrm{AJ}-5$. Doxorubicin was included as a positive control for caspase- 8 and -9 assays at the $\mathrm{I}_{50}$ concentration $(0.5 \mu \mathrm{M})$ established in the cell lines tested. Graphs represent the mean fold change of caspase activity \pm SEM pooled from three independent repeats. Data were analysed using GraphPad Prism 6.0 and a parametric unpaired $t$-test was performed where ${ }^{*} p<0.05,{ }^{* *} p<0.01,{ }^{* * *} p<0.001$

period than cisplatin in these RMS cells. Furthermore, we show that AJ-5 displays a selectivity index of $\geq 2$, which suggests that it has specificity for RMS cells ${ }^{47,48}$. Indeed, whereas AJ-5 treated RMS cells were unable to form any colonies, mouse myoblasts treated with 1.5-3 times higher concentrations formed colonies with areas comparable to that obtained for vehicletreated cells (Fig. 2a). Furthermore, mesenchymal stem cells from different sources are thought to represent the target cell of origin for a variety of human sarcomas and our cell viability assays (Fig. 1c) show that the A100021501 mesenchymal stem cells were the most sensitive nonmalignant cell line to AJ-5 treatment ${ }^{20}$. This suggests that AJ-5 may have the ability to selectively target the sarcoma-initiating cell which could prove to be advantageous and more efficacious than current therapies. Taken together these findings support evidence that, compared to platinum compounds, palladium complexes display superior anti-cancer activity and that they may associate with fewer side effects.

While tumours initially respond to proapoptotic therapies, they frequently acquire the ability to bypass the apoptotic pathway and develop drug resistance leading to tumour recurrence ${ }^{49,50}$. It is thus predicted that drugs that can trigger alternative or multiple programmed cell death (PCD) pathways such as autophagy (PCD type II) and programmed necrosis (PCD type III) would be more efficacious $^{51}$. The annexin V-FITC assays in Fig. 4c reveal that AJ-5 induces not only a significant apoptotic population but also a significant cell population which could represent necrosis, programmed necrosis (necroptosis) and/or secondary necrosis which occurs when there is insufficient clearance of apoptotic cells ${ }^{49,52}$. Interestingly, we have preliminary western blot data that show that AJ-5 treatment causes an increase in phosphorylated receptorinteracting serine/threonine protein kinase 3 (RIP3), a critical component of necroptosis, as well as increased levels of active (phosphorylated) mixed lineage kinase domain-like protein (MLKL), a key executioner of necroptosis (data not shown). The possibility that AJ-5 may induce necroptosis is exciting; however, to confirm this annexin V-FITC assays of AJ-5-treated cells in the presence of necrostatin-1 and the pan-caspase inhibitor Z-VAD-fmk will need to be performed.

Here we show for the first time that AJ-5 reduces autophagic flux in both aRMS and eRMS cells. This is an important finding because autophagy has been reported to promote cell survival and chemotherapeutic drug resistance in a number of sarcomas, including RMS, and thus autophagy has been identified as a drug target to treat sarcomas ${ }^{50,51,53}$. For example, the autophagy inhibitor, chloroquine, enhanced RMS cell death induced by ciclopirox olamine, bortezomib and 17-DMAG and stimulation of autophagy by rapamycin prevented this ${ }^{54,55}$. Consistent with our findings, palladium nanoparticles have recently been shown to block autophagic flux in Hela cells as shown by increased LC3II/I ratios, reduced degradation of p62 and autophagosome accumulation ${ }^{56}$. It is important to note that AJ-5 induces autophagy PCD in melanoma and breast cancer cells, which suggests that the effect of palladium complexes on autophagy may be cancer type specific.

In conclusion, we show that AJ-5 may be a promising chemotherapeutic to treat aRMS and eRMS because it displays potent and selective cytotoxicity at submicromolar concentrations and inhibits the ability of RMS cells to survive, proliferate and migrate. Importantly, AJ-5 induces apoptosis and reduces autophagic flux and it displays a favourable pharmacokinetic and safety profile.

\section{Materials and methods \\ Cell culture}

RH30 human aRMS cells (kindly provided by Associate Professor Judith Davie, Southern Illinois University), AX-OH-1 human aRMS and FL-OH-1 human eRMS cells (kindly provided by Professor Stefan Bath, University of Cape Town) were cultured in Roswell Park Memorial Institute Medium (RPMI)-1640 (Sigma Aldrich, Missouri, USA). RD human eRMS cells (ATCC $^{\oplus}$ CCL-136 $^{\mathrm{m}}$ ), FG0 and DMB human skin fibroblasts (kindly provided by Associate Professor Denver Hendricks, University of Cape Town), mouse myoblast 
A

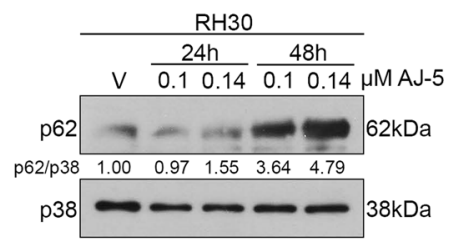

B

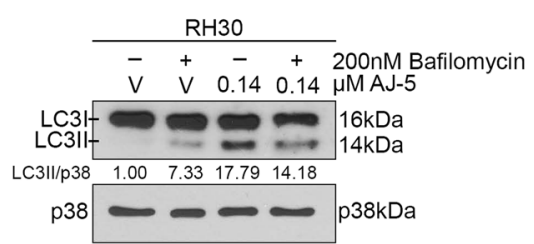

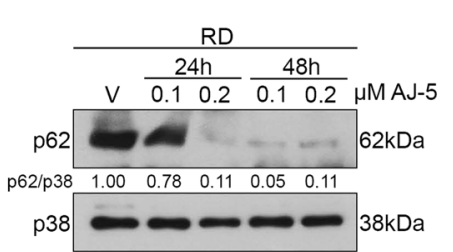

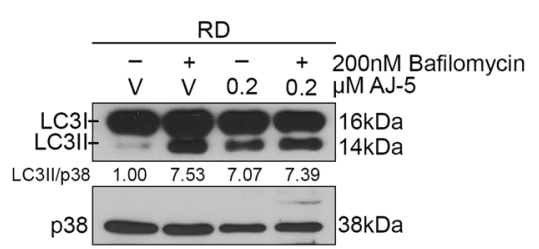

C

RD
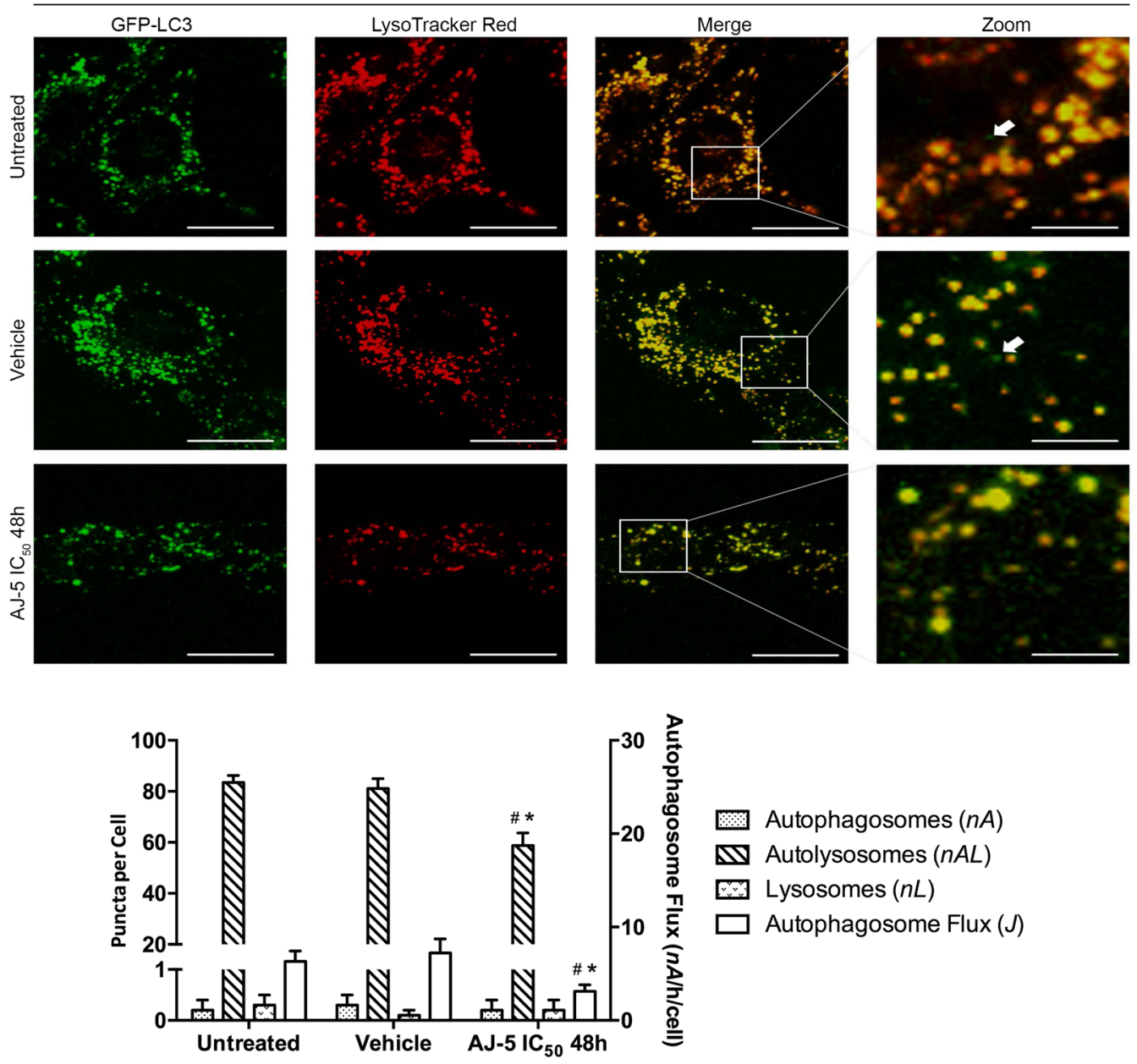

Fig. 6 AJ-5 reduces autophagic flux in RD and RH30 cells. a Western blotting of p62/SQSTM1 protein levels in RH30 and RD cells treated with vehicle $(\mathrm{V}), 0.1 \mu \mathrm{M}$ or $\mathrm{IC}_{50} \mathrm{AJ}-5$ for 24 and $48 \mathrm{~h}$. b Western blotting showing LC3I and LC3Il protein levels in RH30 and RD cells treated with vehicle (V) or $\mathrm{IC}_{50} \mathrm{AJ}-5$ for $24 \mathrm{~h}$ followed by $2 \mathrm{~h}$ of treatment with $200 \mathrm{nM}$ bafilomycin A1. For western blots, $\mathrm{p} 38$ was used as a loading control and densitometry readings were obtained using ImageJ. Protein expression levels are represented as a ratio of protein of interest/p38 normalized to vehicle control sample. Blots are representative of at least two independent repeats. c Representative single-cell fluorescence maximum intensity projection micrographs ( $\times 630$; Carl Zeiss LSM 780; scale bar is $20 \mu \mathrm{M}$ ) and pool size quantification of autophagy pathway intermediates: autophagosomes (GFP$L C 3, n A$ ) (indicated with white arrows in the merged image), autolysosomes (LysoTracker Red, $n A L$ ) and lysosomes (merged, $n L$ ). Autophagosome flux J was calcuclated. Data were analysed using GraphPad Prism 6.0 and a parametric unpaired t-test was performed ${ }^{*} p<0.05,{ }^{* *} p<0.01,{ }^{* * *} p<0.001$. \# compared to untreated control, * compared to vehicle control 


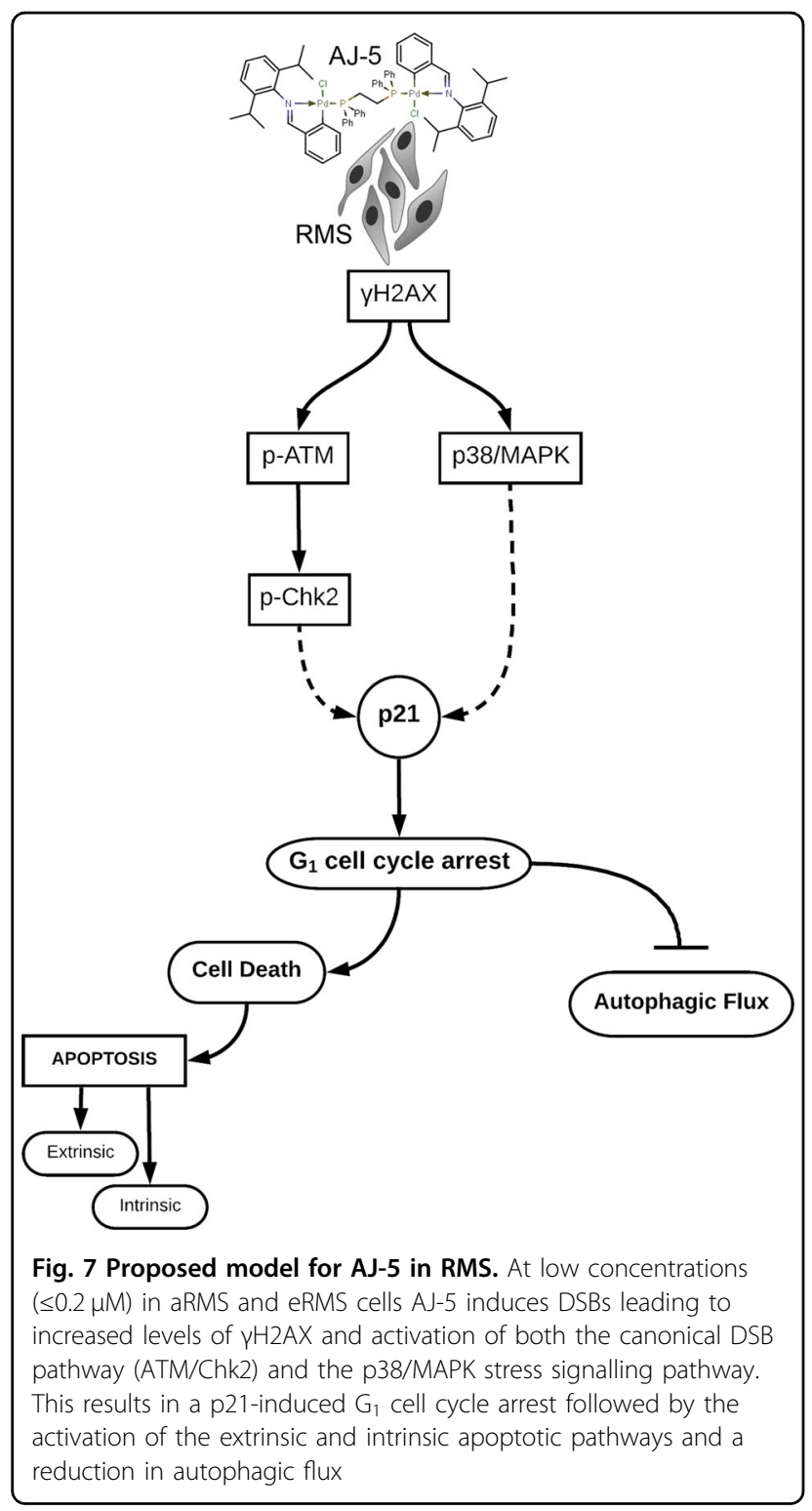

cells (ATCC ${ }^{\circledast}$ CRL-1772 $2^{\text {rm }}$ ), human mesenchymal stem cells A10021501 (kindly provided by Professor Michael Pepper, University of Pretoria and confirmed to meet the criteria to be defined as mesenchymal stem cells as set out by the Mesenchymal and Tissue Stem Cell Committee of the International Society for Cellular Therapy $^{57}$ ), HT1080 human fibrosarcoma cells (ATCC ${ }^{\circ}$ CCL-120 ${ }^{\text {mex }}$ ), SW1353 human chondrosarcoma cells (ATCC ${ }^{\oplus}$ HTB-94 $\left.{ }^{\mathrm{mm}}\right)$, SW982 human synovial sarcoma cells $\left(\right.$ ATCC $^{\circ}$ HTB-93 $^{\mathrm{mm}}$ ), SW872 human liposarcoma (ATCC ${ }^{\circ}$ HTB-92 $^{\mathrm{m \omega}}$ ) and MG-63 human osteosarcoma cells (kindly provided by Associate Professor Philippa Hulley, University of Oxford) were cultured in Dulbecco's modified Eagle's medium (Sigma Aldrich). All culture medium was supplemented with $10 \%$ heat-inactivated foetal bovine serum, $100 \mathrm{U} / \mathrm{mL}$ penicillin and $100 \mu \mathrm{g} / \mathrm{mL}$ streptomycin. Cells were maintained at $37{ }^{\circ} \mathrm{C}$ in a $95 \%$ air and $5 \% \mathrm{CO}_{2}$ humidified incubator. Medium was replaced every 2 to 3 days and cells were routinely subjected to mycoplasma tests. Only mycoplasma free cells were used in experiments. Cell morphology was monitored using an Olympus CKX41 inverted microscope (MSAC Ltd, UK) and imaged with an $\mathrm{EVOS}^{\mathrm{m}} \mathrm{XL}$ AMEX1000 Core Imaging System (Thermo Fisher Scientific, Massachusetts, USA).

\section{Cell treatments}

AJ-5, synthesized as previously described ${ }^{18}$, was dissolved in dimethyl sulfoxide (DMSO) (D8418; Sigma Aldrich) to give a $5 \mathrm{mM} \mathrm{AJ}-5$ stock which was stored at room temperature (RT) and used within 5 days. AJ-5 was diluted in cell culture medium to achieve the desired final concentration and a vehicle control (DMSO) of the same concentration was prepared simultaneously. Cells were treated at a confluency of $60 \%$. For western blot experiments to determine autophagy flux, cells were treated with $200 \mathrm{nM}$ bafilomycin A1 (B1793; Sigma Aldrich) for 2 $\mathrm{h}$ post AJ-5 treatment. Doxorubicin (z/26/0167; Teva Pharmaceutical Industries Ltd, Israel) was used as a positive control in Caspase-Glo experiments at a concentration of $0.5 \mu \mathrm{M}$.

\section{Cell viability assays}

Cells were seeded in 96-well plates and treated the next day with a range of AJ-5 concentrations $(0.1-1.0 \mu \mathrm{M})$ or vehicle $(1.0 \mu \mathrm{M}$ DMSO $)$ for $48 \mathrm{~h}$. Cell viability was measured using the 3-(4,5-dimethylthiazol-2-yl)-2,5-diphenyltrazolium bromide (MTT) assay (11465007001; Sigma Aldrich) according to the manufacturer's instructions. Mean cell viability was calculated as a percentage of the mean vehicle control. At least three independent experiments in quadruplicate were performed from which the half maximal inhibitory concentration $\left(\mathrm{IC}_{50}\right)$ was determined using GraphPad Prism version 6.0 (GraphPad Software, California, USA). The selectivity index (SI) was determined by dividing the $\mathrm{IC}_{50}$ of a normal cell line by the $\mathrm{IC}_{50}$ of a cancer cell line.

\section{Clonogenic assays}

Cells were seeded in 6-well plates and treated the following day with $\mathrm{IC}_{50}, 1 / 2 \mathrm{IC}_{50}, 1 / 4 \mathrm{IC}_{50} \mathrm{AJ}-5$ and vehicle. After $24 \mathrm{~h}, 800-4000$ cells were seeded in $35 \mathrm{~mm}$ dishes in drug-free medium. Formation of colonies were monitored and after 7-21 days cells were fixed with 3:1 methanol: acetic acid and stained with $0.5 \%$ crystal violet (Sigma Aldrich) in 100\% methanol. Colonies were imaged and percentage colony area was determined using Image) $\mathrm{v} .50 \mathrm{i}^{58}$ and the plugin ColonyArea ${ }^{59}$. Colony area was 
determined for each drug concentration and expressed as a percentage of vehicletreated control.

\section{Scratch motility assay}

Cells were seeded in 6-well plates and treated with $\mathrm{IC}_{50}$ AJ-5 and vehicle for $24 \mathrm{~h}$. Cells were then collected, counted and replated to achieve $100 \%$ confluency in a $24-$ well plate. The following day a sterile $2 \mu \mathrm{L}$ pipette tip was used to make a vertical scratch in the cell monolayer of each well and the cells treated with $10 \mu \mathrm{M}$ mitomycin $\mathrm{C}$ (M4287; Sigma Aldrich) to inhibit proliferation. Cells were imaged at $0,3,6,9,12$ and $24 \mathrm{~h}$ post wound formation and ImageJ v1.50i ${ }^{58}$ was used to calculate the area of the scratch. The total areas migrated was determined by subtracting the area for a specific time point from the area measured at $0 \mathrm{~h}$.

\section{Western blot analyses}

Cells were harvested after exposure to $\mathrm{IC}_{50} \mathrm{AJ}-5,0.1$ $\mu \mathrm{M} \mathrm{AJ}-5$ or vehicle (DMSO) for 24 and $48 \mathrm{~h}$. Cells were lysed at $4{ }^{\circ} \mathrm{C}$ in whole-cell lysis buffer $(0.125 \mathrm{M}$ Tris- $\mathrm{HCl}$ $\mathrm{pH} 6.8,4 \%$ SDS, $0.2 \%$ glycerol, $0.1 \% \beta$-mercaptoethanol and a pinch of bromophenol blue) and boiled for $10 \mathrm{~min}$. Proteins were resolved by SDS-PAGE $(6-15 \%$ gels) and transferred to Hybond ECL membranes (Amersham, UK). The membranes were incubated with primary antibodies: rabbit polyclonal antibodies to phospho-histone H2A.X (Ser139) (\#2577), phosphochk2 (Thr68) (\#221), phospho-p38 MAPK (Thr180/ Tyr182) (\#9211), cleaved caspase-3 (Asp175) (\#9661), PARP (\#9542), caspase-9 (\#9502), LC3B (\#2775), p38 MAPK (\#9212), rabbit monoclonal antibody to cleaved caspase-7 (Asp198) (D6H1) (\#8438), mouse monoclonal antibodies to phospho-ATM (Ser1981) (D6H9) (\#5883), Cyclin B1 (V152) (\#4135), Caspase-8 (1C12) (\#9746), SQSTM1/p62 (D5L7G) (\#88588) from Cell Signaling Technology (Massachusetts, USA); mouse monoclonal antibody to p53 (DO-1) (sc-126), rabbit polyclonal antibodies to p21 (C-19) (sc-397), cyclin A (H-432) (sc751), cyclin B1 (H-433)(sc-752), PARP-1 (H-250) (sc7150) from Santa Cruz Biotechnology (Texas, USA); rabbit polyclonal antibody to p38 MAP kinase (M0800) from Sigma Aldrich. After primary antibody incubation, membranes were incubated with goat anti-rabbit or goat anti-mouse HRP-conjugated secondary antibodies (BioRad Laboratories, California, USA). Antibody reactive proteins were visualized by enhanced chemiluminescence using SuperSignal West Pico Chemiluminescent Substrate Kit (Thermo Fisher Scientific) or WesternBright ECL HRP Substrate Kit (Advansta, California, USA). Densitometry readings were obtained using Image $\mathrm{v} 1.50 \mathrm{i}^{58}$ and protein expression levels were represented as a ratio of protein of interest/p38 loading control normalized to the vehicletreated control sample where appropriate. All blots are representative of at least two independent repeats.

\section{Immunofluorescence}

Cells were plated on glass coverslips and treated with $\mathrm{IC}_{50} \mathrm{AJ}-5$ or vehicle. Cells were fixed with ice-cold $100 \%$ methanol at $-20^{\circ} \mathrm{C}$ for $5 \mathrm{~min}$ followed by blocking and permeabilization with $0.2 \%$ Triton-X-100/5\% bovine serum albumin in phosphate-buffered saline (PBS) for 30 min at RT. Slides were incubated with antibodies against phospho-histone H2A.X (\#2577) (1:500) or LC3B (\#2775) (1:200) (Cell Signaling Technology) in blocking buffer overnight at $4{ }^{\circ} \mathrm{C}$. After PBS washes coverslips were incubated with donkey anti-rabbit or donkey anti-mouse Cy3-conjugated secondary antibodies (Jackson ImmunoResearch Laboratories Inc., Pennsylvania, USA) diluted 1:1000 for $1.5 \mathrm{~h}$ in blocking buffer at RT. A secondaryantibody-only control was always included in these experiments. Cells were then counterstained with Hoechst (33342; Invitrogen, California, USA) for $10 \mathrm{~min}$ at RT in the dark, washed with PBS and then coverslips were mounted onto glass slides using Mowiol mounting medium. Cells were imaged with an LSM 510 confocal microscope (Carl Zeiss, Germany) using a PlanApochromat $\times 63 / 1.40$ oil DIC objective. Multiple z layers were acquired with $1 \mu \mathrm{M}$ step width. Images were processed using ZEN 2012 imaging software (Carl Zeiss) and maximum intensity projections were generated. For quantification, mean fluorescence was measured from at least 20 fields of view per treatment condition and pooled from three independent repeats.

\section{Cell cycle analysis}

Cells were seeded and treated the following day with $\mathrm{IC}_{50} \mathrm{AJ}-5$ and vehicle for 24 and $48 \mathrm{~h}$, trypsinized, washed with PBS, counted and permeabilized in $70 \% \mathrm{EtOH}$ at $-20^{\circ} \mathrm{C}$ overnight. Cells were pelleted and treated with 50 $\mu \mathrm{g} / \mathrm{mL}$ RNase (Fermentas, Massachusetts, USA) in PBS at $37^{\circ} \mathrm{C}$ for $15 \mathrm{~min}$ and then stained with propidium iodide (PI). A minimum of 50,000 cells/sample were subjected to analysis using a Becton Dickinson FACSCalibur flow cytometer (Becton Dickinson, New Jersey, USA) with a $488 \mathrm{~nm}$ coherent laser. The data were acquired using CellQuest Pro version 5.2.1. software (Becton Dickinson) and the analyses were done using ModFit version 2.0. software (Verity Software House Inc, Maine, USA).

\section{Annexin V-FITC assays}

Cells were seeded, trypsinized, washed and counted as described for cell cycle analysis. The FITC Annexin V/ Dead Cell Apoptosis kit (V13242; Thermo Fisher Scientific) was used according to the manufacturer's instructions. A negative control of unstained cells and positive controls of cells treated with $3 \%$ formaldehyde for $30 \mathrm{~min}$ 
on ice and stained with either PI or Annexin V were used to gate the flow cytometer for viable, early apoptotic, late apoptotic, and necrotic cell populations. Samples were subjected to FACS analyses as described for cell cycle analysis. Data were obtained and analysed using CellQuest Pro version 5.2.1. software.

\section{Caspase activity assays}

Cells were seeded and treated for $24 \mathrm{~h}$ with $\mathrm{IC}_{50} \mathrm{AJ}-5$, vehicle or $0.5 \mu \mathrm{M}\left(\mathrm{IC}_{50}\right)$ doxorubicin which was included and as a positive control. Cells were trypsinized, washed and counted and caspase- $8,-9$, and $-3 / 7$ activity was measured using the appropriate Caspase- $\mathrm{Glo}^{\oplus}$ assay kits (Promega, Wisconsin, USA) according to the manufacturer's instructions. Luminescence was measured 45 min after incubation with Caspase $\mathrm{Glo}^{\circledR}$ reagent using a Luminoscan Ascent luminometer (Thermo LabSystems Inc., Massachusetts, USA). Luminescence signal for treated samples were normalized to vehicle-treated samples to express a fold change increase in caspase activity.

\section{Supravital staining with acridine orange}

Cells were seeded on glass coverslips and treated for 24 $\mathrm{h}$ with $\mathrm{IC}_{50} \mathrm{AJ}-5$ or vehicle and then stained with $2 \mu \mathrm{g} / \mathrm{mL}$ acridine orange (Michrome No. 714; Edward Gurr Ltd, UK), which was added directly to the medium, for 15 min at RT in the dark. Coverslips were mounted on glass slides and visualized and imaged as described for immunofluorescence.

\section{Single-cell autophagosome flux analyses}

The autophagy steady-state variables were measured in $\mathrm{RD}$ cells treated with $\mathrm{IC}_{50}$ AJ-5 for $48 \mathrm{~h}$. The autophagosome flux was calculated from the rate of autophagosome accumulation as previously described ${ }^{60}$. Briefly, this was determined after the complete inhibition of fusion between autophagosomes and lysosomes using $400 \mathrm{nM}$ bafilomycin A1 (B0025; LKT Laboratories Inc., Minnesota, USA).

Cells were transfected with a GFP-LC3 construct using Lipofectamine 3000 (L3000001; Thermo Fisher Scientific) according to the manufacturer's instructions and maintained for 2 days in order to achieve high levels of GFPLC3 expression. Thereafter, cells were harvested by trypsinization and seeded into an eight-chamber coverslip-based dish (155411 Nunc ${ }^{\mathrm{mm}}$ Lab-Tek ${ }^{\mathrm{Tm}}$; Thermo Fisher Scientific) to achieve $60 \%$ confluency after which they were treated with $\mathrm{IC}_{50} \mathrm{AJ}-5$ for $48 \mathrm{~h}$.

Autophagosome, autolysosome and lysosome pool size was assessed by supplementing the culture media with 25 nM LysoTracker Red (L7528; Thermo Fisher Scientific) 2 $\mathrm{h}$ prior to image acquisition. An LSM 780 confocal with ELYRA S.1 Superresolution platform (Carl Zeiss) was used for image acquisition. The eight-chamber dish was placed in a heated chamber maintained at $5 \% \mathrm{CO}_{2}$ that encased the microscope objective. An LCI PlanApochromat $\times 63 / 1.4$ oil DIC M27 objective was used and samples were excited with an Argon multiline laser $25 \mathrm{~mW}$ at 488 and $514 \mathrm{~nm}$ with appropriate beam splitters and GaAsP detector 32+2 PMT. Multiple $\mathrm{z}$ layers were acquired with $1 \mu \mathrm{M}$ step width. Laser power and electron gain were set to achieve an optimal signal to noise ratio without causing photo-toxicity. Images were processed using ZEN 2011 imaging software (Carl Zeiss) and the maximum intensity projections were exported to Fiji software $^{61}$ for further analysis ${ }^{60}$.

\section{Pharmacokinetics studies of AJ-5 in healthy mice}

All studies and procedures were conducted with prior approval of the Animal Ethics Committee of the University of Cape Town (Protocol 012/049) in accordance with the South African National Standard (SANS 10386:008) for the Care and Use of Animals for Scientific Purposes $^{62}$, and guidelines from the Department of Health ${ }^{63}$.

Pharmacokinetic parameters of AJ-5 were determined after IV, IP and PO dosing to MF1 nude mice randomly divided into groups. AJ-5 was prepared in the respective formulations immediately prior to dosing. For the IV group, the compound was made up in a mixture of $10 \%$ DMSO and $90 \%$ of an IV mixture, which consisted of $60 \%$ propylene glycol, $10 \%$ ethanol and 30\% polyethylene glycol. For the PO group, AJ-5 was dissolved in DMSO and a hydroxypropylmethylcellulose solution $(0.5 \%$ in water $)$ containing $0.2 \%$ Tween 80 in a ratio of 1:9. For the IP group, AJ-5 was dissolved in 10\% DMSO and $90 \%$ phosphate buffer solution. Mice were permitted access to food and water ad libitum. AJ-5 was administered $(100 \mu \mathrm{L})$ by oral gavage at a dose of $20 \mathrm{mg} / \mathrm{kg}(n=3)$, intravenously into the penile dorsal vein at a dose of $2 \mathrm{mg} / \mathrm{kg}(n=2)$ and intraperitoneally at a dose of $2 \mathrm{mg} / \mathrm{kg}(n=3)$.

Blood samples ( $20 \mu \mathrm{L}$ per sample) were collected by tail tip bleeding in lithium heparin tubes at predetermined time points to evaluate the kinetic profile over $24 \mathrm{~h}$. Samples were stored at $-80^{\circ} \mathrm{C}$ until analysis. Blood samples together with calibration standards and quality controls were quantified using inductively coupled plasma mass spectrometry. All results reported are for palladium (Pd). Pharmacokinetic parameters of the AJ-5 complex were determined using non-compartmental analysis in PK Solutions 2.0 (Summit Research Systems, Montrose CO, USA).

\section{Statistical analyses}

All data were obtained from at least three independent experiments (unless otherwise stated) with error bars representing standard error of the mean (SEM). Data were analysed using GraphPad Prism version 6.0 (GraphPad 
Software) and a parametric unpaired $t$-test was performed. Significance was accepted at $* p<0.05,{ }^{* * *} p<0.01$ and $* * * 0.001$.

\section{Acknowledgements}

This work was supported by grants from the SA Medical Research Council, the National Research Foundation (NRF), Cancer Association of South Africa (CANSA) and the University of Cape Town. The content is solely the responsibility of the authors and does not necessarily represent the official views of the funding agencies. We would like to thank Associate Professor Judith Davie, Professor Stefan Bath, Associate Professor Denver Hendricks, Professor Michael Pepper and Associate Professor Philippa Hulley who kindly provided us with cell lines used in this study.

\section{Author details}

'Division of Cell Biology, Department of Human Biology, Faculty of Health Sciences, University of Cape Town, Cape Town, Western Cape, South Africa. ${ }^{2}$ Department of Physiological Sciences, Stellenbosch University, Stellenbosch, Western Cape, South Africa. ${ }^{3}$ Division of Clinical Pharmacology, Department of Medicine, Faculty of Health Sciences, University of Cape Town, Cape Town, Western Cape, South Africa. ${ }^{4}$ Department of Chemistry and Polymer Science, Stellenbosch University, Stellenbosch, Western Cape, South Africa

\section{Conflict of intererst}

The authors declare that they have no conflict of interest.

\section{Publisher's note}

Springer Nature remains neutral with regard to jurisdictional claims in published maps and institutional affiliations.

The online version of this article (https://doi.org/10.1038/s41420-019-0139-9) contains supplementary material, which is available to authorized users.

Received: 20 November 2018 Revised: 19 December 2018 Accepted: 2 January 2019

Published online: 28 January 2019

\section{References}

1. Stout, A. P. Rhabdomyosarcoma of the skeletal muscles. Ann. Surg. 123, 447-472 (1946).

2. Dagher, R. \& Helman, L. Rhabdomyosarcoma: an overview. Oncologist 4, 34-44 (1999).

3. Perez, E. A. et al. Rhabdomyosarcoma in children: a SEER population based study. J. Surg. Res. 170, e243-e251 (2011).

4. Wachtel, M. et al. Subtype and prognostic classification of rhabdomyosarcoma by immunohistochemistry. J. Clin. Oncol. 24, 816-822 (2006).

5. Ward, E., DeSantis, C., Robbins, A., Kohler, B. \& Jemal, A. Childhood and adolescent cancer statistics, 2014. CA Cancer J. Clin. 64, 83-103 (2014).

6. Egas-Bejar, D. \& Huh, W. W. Rhabdomyosarcoma in adolescent and young adult patients: current perspectives. Adolesc. Health Med. Ther. 5, 115-125 (2014).

7. Hosoi, H. Current status of treatment for pediatric rhabdomyosarcoma in the USA and Japan. Pediatr. Int. 58, 81-87 (2016).

8. Pappo, A. S., Shapiro, D. N., Crist, W. M. \& Maurer, H. M. Biology and therapy of pediatric rhabdomyosarcoma. J. Clin. Oncol. 13, 2123-2139 (1995).

9. Neidle, S. Cancer Drug Design and Discovery (Academic Press, San Diego, 2013).

10. Makin, G. Principles of chemotherapy. Paediatr. Child Health (Oxf.) 24, 161-165 (2014).

11. Silverman, R. B. \& Holladay, M. W. The Organic Chemistry of Drug Design and Drug Action (Academic Press, San Diego, 2014).

12. Smith, H. J. \& Williams, H. Smith and Williams' Introduction to the Principles of Drug Design and Action 4th edn (CRC Press Taylor \& Francis Group, Boca Raton, 2005).

13. Ndagi, U., Mhlongo, N. \& Soliman, M. E. Metal complexes in cancer therapyan update from drug design perspective. Drug Des. Dev. Ther. 11, 599-616 (2017).
14. Ulukaya, E. et al. Anti-cancer activity of a novel palladium(II) complex on human breast cancer cells in vitro and in vivo. Eur. J. Med. Chem. 46, 4957-4963 (2011).

15. Kruszewski, M. et al. Differential toxic effect of cis-platinum(II) and palladium(II) chlorides complexed with methyl 3,4-diamine-2,3,4,6-tetradeoxy-alpha-L-lyxohexopyranoside in mouse lymphoma cell lines differing in DSB and NER repair ability. Teratog. Carcinog. Mutagen. 1(Suppl), 1-11 (2003).

16. Akdi, K. et al. Study of the biological effects and DNA damage exerted by a new dipalladium-Hmtpo complex on human cancer cells. J. Inorg. Biochem. 90, 51-60 (2002).

17. Carreira, M., Calvo-Sanjuán, R., Sanaú, M., Marzo, I. \& Contel, M. Organometallic palladium complexes with a water-soluble iminophosphorane ligand as potential anticancer agents. Organometallics 31, 5772-5781 (2012).

18. Aliwaini, S., Swarts, A. J., Blanckenberg, A., Mapolie, S. \& Prince, S. A novel binuclear palladacycle complex inhibits melanoma growth in vitro and in vivo through apoptosis and autophagy. Biochem. Pharmacol. 86, 1650-1663 (2013).

19. Aliwaini, S. et al. The palladacycle, AJ-5, exhibits anti-tumour and anti-cancer stem cell activity in breast cancer cells. Cancer Lett. 357, 206-218 (2015).

20. Rodriguez, R., Rubio, R. \& Menendez, P. Modeling sarcomagenesis using multipotent mesenchymal stem cells. Cell Res. 22, 62-77 (2012).

21. Hettmer, S. \& Wagers, A. J. Muscling in: uncovering the origins of rhabdomyosarcoma. Nat. Med. 16, 171-173 (2010).

22. Galluzzi, L. et al. Guidelines for the use and interpretation of assays for monitoring cell death in higher eukaryotes. Cell Death Differ. 16, 1093-1107 (2009).

23. Valdiglesias, V., Giunta, S., Fenech, M., Neri, M. \& Bonassi, S. үH2AX as a marker of DNA double strand breaks and genomic instability in human population studies. Mutat. Res. 753, 24-40 (2013).

24. Sionov, R. V. \& Haupt, Y. The cellular response to p53: the decision between life and death. Oncogene 18, 6145-6157 (1999).

25. Felix, C. A. et al. Frequency and diversity of p53 mutations in childhood rhabdomyosarcoma. Cancer Res. 52, 2243-2247 (1992).

26. Karimian, A.r Ahmadi, Y. \& Yousefi, B. Multiple functions of p21 in cell cycle, apoptosis and transcriptional regulation after DNA damage. DNA Repair (Amst.). 42, 63-71 (2016).

27. Soldani, C. \& Scovassi, A. I. Poly(ADP-ribose) polymerase-1 cleavage during apoptosis: an update. Apoptosis 7, 321-328 (2002).

28. Oliver, F. J. Importance of poly(ADP-ribose) polymerase and its cleavage in apoptosis. Lesson from an uncleavable mutant. J. Biol. Chem. 273 33533-33539 (1998).

29. Wang, Q., Na, B., Ou, J. J., Pulliam, L. \& Yen, T. S. B. Hepatitis B virus alters the antioxidant system in transgenic mice and sensitizes hepatocytes to Fas signaling. PLOS ONE 7, e36818 (2012).

30. Hoffmann, J. C., Pappa, A., Krammer, P. H. \& Lavrik, I. N. A new C-terminal cleavage product of procaspase-8, p30, defines an alternative pathway of procaspase-8 activation. Mol. Cell. Biol. 29, 4431-4440 (2009).

31. Pankiv, S. et al. p62/SQSTM1 binds directly to Atg8/LC3 to facilitate degradation of ubiquitinated protein aggregates by autophagy. J. Biol. Chem. 282, 24131-24145 (2007)

32. Mauvezin, C. \& Neufeld, T. P. Bafilomycin A1 disrupts autophagic flux by inhibiting both V-ATPase-dependent acidification and Ca-P60A/SERCAdependent autophagosome-lysosome fusion. Autophagy 11, 1437-1438 (2015).

33. Wang, C. Childhood rhabdomyosarcoma: recent advances and prospective views. J. Dent. Res. 91, 341-350 (2012).

34. Breneman, J. C. et al. Prognostic factors and clinical outcomes in children and adolescents with metastatic rhabdomyosarcoma - a report from the Intergroup Rhabdomyosarcoma Study IV. J. Clin. Oncol. 21, 78-84 (2003).

35. Malempati, S. \& Hawkins, D. S. Rhabdomyosarcoma: review of the Children's Oncology Group (COG) Soft-Tissue Sarcoma Committee experience and rationale for current COG studies. Pediatr. Blood Cancer 59, 5-10 (2012).

36. Stevens, M. C. G. Treatment for childhood rhabdomyosarcoma: the cost of cure. Lancet Oncol. 6, 77-84 (2005).

37. Oberlin, O. et al. Prognostic factors in metastatic rhabdomyosarcomas: results of a pooled analysis from United States and European cooperative groups. J. Clin. Oncol. 26, 2384-2389 (2008).

38. Dasgupta, R. \& Rodeberg, D. A. Update on rhabdomyosarcoma. Semin. Pediatr. Surg. 21, 68-78 (2012). 
39. McDowell, H. P. et al. Outcomes in paediatric metastatic rhabdomyosarcoma: results of The International Society of Paediatric Oncology (SIOP) study MMT98. Eur. J. Cancer 46, 1588-1595 (2010).

40. Perkins, S. M., Shinohara, E. T., DeWees, T. \& Frangoul, H. Outcome for children with metastatic solid tumors over the last four decades. PLOS ONE 9, e100396 (2014).

41. Shern, J. F. et al. Comprehensive genomic analysis of rhabdomyosarcoma reveals a landscape of alterations affecting a common genetic axis in fusionpositive and fusion-negative tumors. Cancer Discov. 4, 216-231 (2014).

42. Holohan, C., Van Schaeybroeck, S., Longley, D. B. \& Johnston, P. G. Cancer drug resistance: an evolving paradigm. Nat. Rev. Cancer 13, 714-726 (2013).

43. Jahromi, E. Z. et al. Palladium complexes: new candidates for anti-cancer drugs. J. Iran. Chem. Soc. 13, 967-989 (2016).

44. Johnstone, T. C., Suntharalingam, K. \& Lippard, S. J. The next generation of platinum drugs: targeted $\mathrm{Pt}(\mathrm{II})$ agents, nanoparticle delivery, and $\mathrm{Pt}(\mathrm{IV})$ prodrugs. Chem. Rev. 116, 3436-3486 (2016).

45. Cocker, H. A., Pinkerton, C. R. \& Kelland, L. R. Characterization and modulation of drug resistance of human paediatric rhabdomyosarcoma cell lines. Br. J. Cancer 83, 338-345 (2000).

46. Cocker, H. A. et al. High levels of the MDM2 oncogene in paediatric rhabdomyosarcoma cell lines may confer multidrug resistance. Br. J. Cancer $\mathbf{8 5}$ 1746-1752 (2001).

47. Badisa, R. B. et al. Selective cytotoxic activities of two novel synthetic drugs on human breast carcinoma MCF-7 cells. Anticancer Res. 29, 2993-2996 (2009).

48. Koch, A., Tamez, P., Pezzuto, J. \& Soejarto, D. Evaluation of plants used for antimalarial treatment by the Maasai of Kenya. J. Ethnopharmacol. 101, 95-99 (2005).

49. Sachet, M., Liang, Y. Y. \& Oehler, R. The immune response to secondary necrotic cells. Apoptosis 22, 1189-1204 (2017).

50. Ricci, M. S. \& Zong, W.-X. Chemotherapeutic approaches for targeting cell death pathways. Oncologist 11, 342-357 (2006).
51. Viola, G. et al. MG-2477, a new tubulin inhibitor, induces autophagy through inhibition of the Akt/mTOR pathway and delayed apoptosis in A549 cells. Biochem. Pharmacol. 83, 16-26 (2012).

52. Silva, M. T. Secondary necrosis: the natural outcome of the complete apoptotic program. FEBS Lett. 584, 4491-4499 (2010).

53. Min, L. et al. Autophagy as a potential target for sarcoma treatment. Biochim Biophys. Acta Rev. Cancer 1868, 40-50 (2017).

54. Zhou, H. et al. Ciclopirox induces autophagy through reactive oxygen speciesmediated activation of JNK signaling pathway. Oncotarget 5, 10140-10150 (2014).

55. Peron, M., Bonvini, P. \& Rosolen, A. Effect of inhibition of the ubiquitinproteasome system and Hsp90 on growth and survival of rhabdomyosarcoma cells in vitro. BMC Cancer 12, 233 (2012).

56. Zhang, $L$. et al. Palladium nanoparticles induce autophagy and autophagic flux blockade in Hela cells. RSC Adv. 8, 4130-4141 (2018).

57. Dominici, M. et al. Minimal criteria for defining multipotent mesenchymal stromal cells. The International Society for Cellular Therapy position statement. Cytotherapy 8, 315-317 (2006).

58. Schneider, C. A., Rasband, W. S. \& Eliceiri, K. W. NIH Image to ImageJ: 25 years of image analysis. Nat. Methods 9, 671-675 (2012).

59. Guzmán, C. et al. ColonyArea: an ImageJ plugin to automatically quantify colony formation in clonogenic assays. PLoS ONE 9, e92444 (2014).

60. du Toit, A., Hofmeyr, J.-H. S., Gniadek, T. J. \& Loos, B. Measuring autophagosome flux. Autophagy 1-12 (2018). https://doi.org/10.1080/15548627.2018. 1469590

61. Schindelin, J. et al. Fiji: an open-source platform for biological-image analysis. Nat. Methods 9, 676-682 (2012).

62. SABS Standards Division. South African Bureau of Standards, South African National Standard: The Care and Use of Animals for Scientific Purposes (SANS 10386:2008) 1st edn (SABS Standards Division, Pretoria, 2015).

63. Department of Health. Ethics in Health Research: Principles, Processes and Structures 2nd edn (Department of Health, Pretoria, 2015). 